\title{
LOCATION ESTIMATION IN WIRELESS AD-HOC SENSOR NETWORKS
}

\author{
Submitted by
}

\section{Enrique Alejandro Gutiérrez Blancarte}

\author{
A thesis submitted to \\ the Faculty of Graduate Studies and Research \\ in partial fulfillment of the requirements for the degree of
}

\section{Master of Applied Science in Electrical Engineering}

\author{
Ottawa-Carleton Institute for Electrical and Computer Engineering \\ Department of Systems and Computer Engineering \\ Carleton University
}

December $19^{\text {th }} 2007$

Copyright @ 2007 Enrique Alejandro Gutiérrez Blancarte 


$\begin{array}{ll}\begin{array}{l}\text { Library and } \\ \text { Archives Canada }\end{array} & \begin{array}{l}\text { Bibliothèque et } \\ \text { Archives Canada }\end{array} \\ \begin{array}{l}\text { Published Heritage } \\ \text { Branch }\end{array} & \begin{array}{l}\text { Direction du } \\ \text { Patrimoine de l'édition }\end{array} \\ \begin{array}{l}\text { 395 Wellington Street } \\ \text { Ottawa ON K1A 0N4 }\end{array} & \begin{array}{l}\text { 395, rue Wellington } \\ \text { Ottawa ON K1A ON4 }\end{array} \\ \text { Canada } & \begin{array}{l}\text { Canada } \\ \end{array}\end{array}$

Yourfile Votre référence ISBN: 978-0-494-36825-1 Ourfile Notre référence ISBN: 978-0-494-36825-1

NOTICE:

The author has granted a nonexclusive license allowing Library and Archives Canada to reproduce, publish, archive, preserve, conserve, communicate to the public by telecommunication or on the Internet, loan, distribute and sell theses worldwide, for commercial or noncommercial purposes, in microform, paper, electronic and/or any other formats.

The author retains copyright ownership and moral rights in this thesis. Neither the thesis nor substantial extracts from it may be printed or otherwise reproduced without the author's permission.
AVIS:

L'auteur a accordé une licence non exclusive permettant à la Bibliothèque et Archives Canada de reproduire, publier, archiver, sauvegarder, conserver, transmettre au public par télécommunication ou par l'Internet, prêter, distribuer et vendre des thèses partout dans le monde, à des fins commerciales ou autres, sur support microforme, papier, électronique et/ou autres formats.

L'auteur conserve la propriété du droit d'auteur et des droits moraux qui protège cette thèse. $\mathrm{Ni}$ la thèse ni des extraits substantiels de celle-ci ne doivent être imprimés ou autrement reproduits sans son autorisation.
In compliance with the Canadian

Privacy Act some supporting forms may have been removed from this thesis.

While these forms may be included in the document page count, their removal does not represent any loss of content from the thesis.
Conformément à la loi canadienne sur la protection de la vie privée, quelques formulaires secondaires ont été enlevés de cette thèse.

Bien que ces formulaires aient inclus dans la pagination, il n'y aura aucun contenu manquant.

\section{Canadä}


The undersigned hereby recommend to

the Faculty of Graduate Studies and Research

acceptance of the thesis

\title{
LOCATION ESTIMATION IN WIRELESS AD-HOC SENSOR NETWORKS
}

\author{
Submitted by \\ Enrique Alejandro Gutiérrez Blancarte \\ in partial fulfillment of the requirements \\ for the degree of Master of Applied Science in Electrical Engineering
}

Dr. Ioannis Lambadaris, Thesis Supervisor

Dr. Victor Aitken, Chair, Department of Systems and Computer Engineering

Carleton University

December $19^{\text {th }}, 2007$ 


\section{Abstract}

Estimating the nodes positions in an ad-hoc sensor network using the information provided by some anchors that know their coordinates is known as the location problem.

We focus our research in two particular schemes for the location problem: rangebased and range-free algorithms. The first category relies on computing pointto-point distance estimates while the second one proposes solutions without the availability of such information. In this thesis, we present an improved hybrid algorithm based on a triangulation scheme. Even though its methodology relies mainly on range-free methods, it provides a refinement phase using ranging measurements. The refinement phase along with our alternative test reduce the location error. Furthermore, the iterative scheme of the algorithm helps to estimate a greater number of nodes.

Simulation results show that the enhanced algorithm has an acceptable performance even when nodes present weak connectivity, a common issue in sparse networks. Furthermore, it is observed from our experiments that the improved algorithm is robust in noisy environments. 


\section{Acknowledgments}

I would like to express my gratitude to my supervisor, Prof. Ioannis Lambadaris, for his guidance during my research.

I am deeply grateful to my family. This thesis would not be possible without the support of my mother, my father and my sister. 


\title{
List of Acronyms
}

\author{
AoA Angle of Arrival \\ APIT Approximate Point-in-Triangle \\ DOI Degree of Irregularity \\ DV-Hop Distance Vector Hop \\ RIM Radio Irregularity Model \\ RMSE Root Mean Square Error \\ RSS Received Signal Strength
}

TDoA Time Difference of Arrival 


\section{Table of Contents}

1 Introduction 1

1.1 Thesis Objectives . . . . . . . . . . . . . . . . . . . . 2

1.2 Contributions and Publications $\ldots \ldots \ldots \ldots$

1.3 Thesis Organization . . . . . . . . . . . . . . . 3

2 Related work 5

2.1 Classification of Location Algorithms . . . . . . . . . . 5

2.1.1 Centralized and Distributed Algorithms ........ 5

2.1.2 Single-hop and Multi-hop Algorithms . . . . . . . . 6

2.1.3 Range-based and Range-free Algorithms . . . . . . . . 9

2.2 The APIT Algorithm: A range-free scheme . . . . . . . . . . 13

2.2 .1 Overview . . . . . . . . . . . . . 13

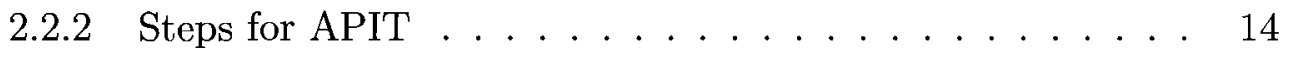

2.2.3 The parameter DOI and the radio model used for APIT . 16

3 A hybrid scheme $\quad 19$

3.1 Drawbacks of the APIT algorithm . . . . . . . . . . . . 19

3.2 The enhanced APIT algorithm . . . . . . . . . . . 21

3.2.1 An Alternative Test: RF profiling . . . . . . . . . . . 21

3.2 .2 Iterative APIT algorithm . . . . . . . . . . . . 23 
3.2.3 Refinement for APIT algorithm . . . . . . . . . . 26

4 Simulation and Performance Analysis 30

4.1 Simulation Objectives $\ldots \ldots \ldots \ldots$

4.2 Simulation Environment and Implementation . . . . . . . . 30

4.3 The General Simulation Setup . . . . . . . . . . . . . . 32

4.3 .1 Location Error Metric . . . . . . . . . . . . . 33

4.4 Performance in sparse networks . . . . . . . . . . . . 33

4.4.1 Simulation Setup for sparse networks . . . . . . . . . 33

4.4.2 Performance of the enhanced algorithm in non noisy environments for sparse networks $\ldots \ldots \ldots$. . . . . . 35

4.4.3 Performance of the enhanced algorithm in noisy environments for sparse networks . . . . . . . . . . . 4 40

4.5 Performance in dense networks $\ldots \ldots \ldots \ldots$

4.5.1 Simulation Setup for dense networks _ . . . . . . . 47

4.5.2 Performance of the enhanced algorithm in non noisy environments for dense networks . . . . . . . . . . . . 5 50

4.5.3 Performance of the enhanced algorithm in noisy environments for dense networks . . . . . . . . . . . . 53

5 Conclusions and Proposed Research 63

5.1 Conclusions . . . . . . . . . . . . . . . 63

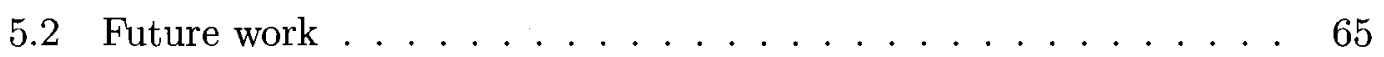

$\begin{array}{ll}\text { References } & 66\end{array}$ 


\section{List of Figures}

2.1 Single-hop Localization . . . . . . . . . . . . . . . . 8

2.2 Multi-hop Localization . . . . . . . . . . . . . . . . . 9

2.3 TDoA Technique . . . . . . . . . . . . . . . 11

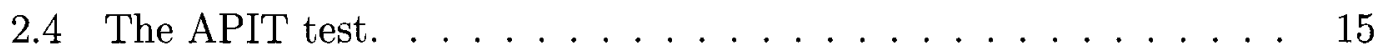

2.5 APIT Aggregation. For each inside decision the counter is increased, otherwise it is decreased. Three triangles are shown, where two of them made inside decisions and the other one made an outside decision $\ldots \ldots \ldots \ldots \ldots \ldots$

2.6 The DOI parameter. Case (a) shows a perfect circular radio model due to the abscense of noise. Case (b) denotes range variations due

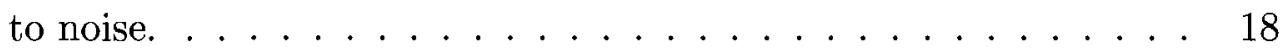

3.1 Drawback on APIT test: Strong condition of connectivity between unknown sensor-anchors and neighborhood-anchors . . . . . . 20

3.2 Anchor $\mathrm{C}$ is not audible by the unknown sensor $a$ whose position can not be estimated . . . . . . . . . . . . . 21 
3.3 An Alternative Test. Triangle with three anchors A,B,C as verteces. Each irregular radio pattern is illustrated around each anchor. In order to decide if $a$ is inside or outside of the triangle, a set of recorded SS measurements and a set of observed measurements of $a$ to A,B,C are needed. Points $1,2,3$ and 4 are some points from the grid in which we can record triplets of SS. . . . . . . . 23

3.4 Iterative APIT. In Case (a), the positions of the unknown sensors are estimated through APIT using the anchors (black nodes). In Case (b), the first set of estimates (gray nodes) and the anchors are used as a new set of anchors. In Case (c), a second set of estimates is obtained (nodes denoted with letter $f$ ) . . . . . 24

3.5 Triangulation in Iterative APIT. A subnetwork $R$ is shown illustrating a set of anchors (black nodes), the first set of estimates (nodes denoted with letter $e$ ), and the second set of estimates (nodes denoted with letter $f$ ) $\ldots \ldots \ldots \ldots$

3.6 A second variant of Iterative APIT $\ldots \ldots \ldots \ldots$

3.7 Refinement procedure . . . . . . . . . . . . . . 29

4.1 Sparse Network. Nodes denoted as ( $)$ are anchors, nodes shown as $(0)$ are unknown sensors. . . . . . . . . . . . 34

4.2 Comparison of the number of located nodes when $(-\bullet-)$ APIT is run only once and (- - ) APIT is run with two iterations. [DOI=0]. 35 
4.3 Normalized RMSE for three cases: $(-\bullet-)$ RMSE computed with the estimates obtained in one iteration, (- - -$)$ RMSE computed with the refined estimates of the 1st iteration, (- $\mathbf{\nabla}-)$ RMSE computed with the refined estimates and estimates of the 2 nd iteration.

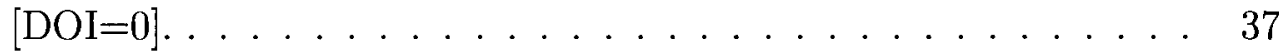

4.4 Normalized RMSE obtained after APIT is run with two iterations. No multilateration scheme is applied. $(-\bullet-)$ RMSE computed with the estimates obtained in two iterations, (- -$)$ RMSE computed with the estimates obtained in the 1st iteration, $(-\mathbf{\nabla}-)$ RMSE computed with the estimates obtained in the 2nd iteration.

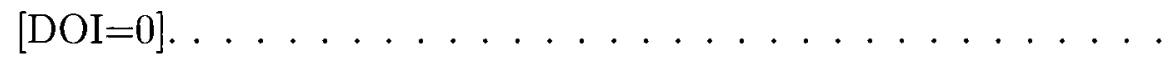

4.5 Normalized RMSE obtained after APIT is run with two iterations and a multilateration scheme as an intermediate step. (- - ) RMSE computed with the refined estimates and estimates of the 2nd iteration, (- - - ) RMSE obtained by running multilateration on initial estimates, $(-\mathbf{\nabla}-)$ RMSE obtained by running APIT in the 2 nd iteration. $[\mathrm{DOI}=0] \ldots \ldots \ldots \ldots \ldots$

4.6 Normalized RMSE for two cases: (- $\bullet$ ) APIT is run with two iterations (- -) APIT is run with two iterations and a multilateration scheme as a final step. $[\mathrm{DOI}=0] \ldots \ldots \ldots \ldots$

4.7 ( - - ) Number of nodes with estimated location for Iterative APIT and (- - ) Number of refined estimates for Iterative APIT.

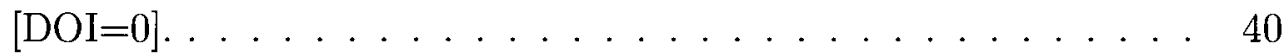

4.8 Comparison of located nodes when $(-\bullet-)$ APIT is run only once and $(-\boldsymbol{\square}-)$ APIT is run with two iterations.[DOI=0.1, DOI $=0.3]$ 
4.9 Comparison of located nodes when $(-\bullet-)$ APIT is run only once and $(-\mathbf{-}-)$ APIT is run with two iterations. [DOI $=0.5$, DOI $=0.7]$

4.10 Normalized RMSE obtained after APIT is run with two iterations. $(-\bullet-)$ RMSE computed with the estimates obtained in two iterations, (- - ) RMSE computed with the estimates obtained in the first iteration, (- - -) RMSE computed with the estimates obtained in the second iteration. [DOI=0.1, DOI=0.3] . . . . 42

4.11 Normalized RMSE obtained after APIT is run with two iterations. $(-\bullet-)$ RMSE computed with the estimates obtained in two iterations, (- - ) RMSE computed with the estimates obtained in the first iteration, (-7-) RMSE computed with the estimates obtained in the second iteration. [DOI=0.5, DOI=0.7] . . . . 43

4.12 The APIT test (a) and the Alternative Test (b) decide if the unknown sensor $a$ is inside or outside triangle A,B,C. Assuming the position of $a$ (c), we can know if $a$ is inside or outside the triangle. 44

$4.13(-\bullet-)$ Ratio of wrong decisions introduced by the APIT test and $(-\mathbf{\nabla}-)$ Ratio of wrong decisions introduced by the Alternative Test when DOI $=0.1 \ldots \ldots \ldots \ldots \ldots \ldots$. . . . . . . . . . 45

$4.14(-\bullet-)$ Ratio of wrong decisions introduced by the APIT test and $(-\mathbf{\nabla}-)$ Ratio of wrong decisions introduced by the Alternative Test when DOI $=0.7 \ldots \ldots \ldots \ldots \ldots \ldots \ldots$

4.15 Refinement applied on APIT in a noisy environment. (- $\bullet$-) Location error obtained by running APIT and (- - -$)$ Location error obtained by running the refinement scheme. $[\mathrm{N}=7] \ldots \ldots 4$

4.16 Dense Network with four regions. Nodes denoted as ( $)$ are anchors, nodes shown as (o) are unknown sensors. . . . . . . . 48 
4.17 Dense Network in which anchors are deployed in each grid point. Nodes denoted as ( $\mathbf{\square})$ are anchors, nodes shown as (o) are unknown

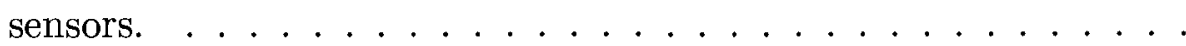

$4.18(-\square-)$ Number of nodes estimated in one iteration and $(-\bullet-)$ Number of nodes estimated in two iterations. $[\mathrm{DOI}=0] \ldots 50$

4.19 Normalized RMSE obtained after APIT is run with two iterations. (- - ) RMSE computed with the estimates obtained in two iterations, (-口-) RMSE computed with the estimates obtained in the first iteration, $(-\boldsymbol{\nabla}-)$ RMSE computed with the estimates obtained in the second iteration. $[\mathrm{DOI}=0] \ldots \ldots 52$

4.20 (- - ) Number of nodes estimated in one iteration and $(-\bullet-)$ Number of nodes estimated in two iterations. [DOI=0.1] . . . . 53

4.21 Normalized RMSE obtained after APIT is run with two iterations. (- - ) RMSE computed with the estimates obtained in two iterations, (- - ) RMSE computed with the estimates obtained in the first iteration, $(-\boldsymbol{\nabla}-)$ RMSE computed with the estimates obtained in the second iteration. $[\mathrm{DOI}=0.1] \ldots \ldots 5$

4.22 (--) Location error using simple APIT and (-) Location error using our iterative scheme. . . . . . . . . . . . . . . . 56

4.23 (--) Ratio of nodes estimates using simple APIT and (-) Ratio of nodes estimates using our iterative scheme. . . . . . . . . 56

4.24 (--) Location error obtained when anchors are distributed randomly and (-) Location error obtained when anchors are deployed deterministically. $[\mathrm{DOI}=0.1] \ldots \ldots \ldots$. . . . . . . 57 
4.25 (--) Ratio of nodes estimates obtained when anchors are distributed randomly and (-) Ratio of nodes estimates when anchors are deployed deterministically. $[\mathrm{DOI}=0.1] \ldots \ldots$. . . . . . 58

4.26 Performance of the tests in a dense network. (- $\bullet$-) Ratio of wrong decisions introduced by the APIT test and (- -$)$ Ratio of wrong decisions introduced by the Alternative Test when DOI=0.1. 59

4.27 Performance of the tests in a dense network. (- $\bullet$-) Ratio of wrong decisions introduced by the APIT test and $(-\boldsymbol{\nabla}-)$ Ratio of wrong decisions introduced by the Alternative Test when DOI=0.7. 61

4.28 Refinement applied on APIT in a noisy environment. (- $\bullet-)$ Location error obtained by running APIT and (- - -) Location error obtained by running the refinement scheme. $[\mathrm{N}=40]$. . . . . 62 


\section{Chapter 1}

\section{Introduction}

Wireless ad-hoc sensor networks with several nodes deployed randomly in an area in which some of them have a priori knowledge of their coordinates (anchors) and the remaining nodes do not have such knowledge (unknown sensors) has been the scenario for studying the sensor localization problem. The solution of this issue constitutes the basis for applications such as monitoring, control, energy-efficient routing, retrieval of sensor data, sending a message to a node at a given location, etc $[1,2]$.

Algorithms have been designed in order to propose a solution for locating the unknown sensors in wireless sensor networks. Thereby, researchers have proposed schemes that lie in two categories: range-based and range-free location algorithms [3]. The first category solves the node location problem through the computation of interdistances while the second one uses techniques that do not rely on such information. It is generally thought that range-free schemes outperform those based on ranging techniques due to the difficult task on finding a method to map physical quantities (e.g. signal strength measurements) into range estimates [2]. On the other hand, range-free algorithms can generate coarse 
localization results [4]. Up to now, it remains questionable which one of the two categories outperforms the other. Since range-free schemes are still promising for location estimation purposes [5], we take them as the basis of our work. However, we further consider techniques based on range estimations that contribute to improving the performance of our algorithm.

We have adopted some of the foundations that the authors in [3] used in their APIT (Approximate Point-in-Triangle) algorithm, which has been shown to provide better results than others $[4,6,7]$.

\section{$1.1 \quad$ Thesis Objectives}

A variety of applications depend on the location estimation of sensors such as habitat monitoring, prevention of disasters, health-care, etc $[1,2]$. Due to this important role, location algorithms have the purpose to estimate the highest possible number of nodes positions spread in a network with acceptable accuracy. The first objective of this dissertation is to propose a location algorithm based on a simple methodology, which can be well-suited for devices with limited resources. The second objective is to implement an hybrid algorithm that obtains the benefits of both range-based and range-free algorithms. The third objective is to evaluate the performance of our localization algorithm in noisy environments. The fourth objective is to test our approach in both sparse and dense networks. Considering the effects of noise and connectivity on networks, we aim to ensure that the algorithm has a reasonably good performance in real scenarios. Finally, the fifth objective is to compare the performance of our approach with a well-known state of the art algorithm. A previously developed method selected for comparison purposes is APIT [3], a range-free algorithm. 


\subsection{Contributions and Publications}

1. We developed a simple and efficient hybrid localization algorithm for wireless ad-hoc sensor networks.

2. The Simulation results showed that our enhanced algorithm is able to estimate the location of a greater number of nodes without significantly increasing the location error introduced by the estimated nodes positions.

3. The algorithm was evaluated in noisy conditions and it showed a reasonably good performance.

Part of the research results have been submitted to the IEEE ICC (International Conference on Communications) 2008.

\subsection{Thesis Organization}

The remaining of this thesis is organized as follows.

Chapter 2 introduces related research for the sensor localization problem and it reviews the methodologies that researchers have proposed for the algorithms on this field. We focus our attention on two categories: range-free and range-based schemes. As a representative algorithm of range-free algorithms, we describe step by step how APIT (Approximate Point-in-Triangle) estimates the positions of unknown sensors in a network.

Chapter 3 describes our proposed hybrid scheme. It stresses the drawbacks of APIT and it proposes specific solutions for each of the drawbacks. It describes in detail, how the enhancements can allow the determination of a greater number of nodes without significantly increasing the location error introduced by the estimated nodes positions. 
The implementation, simulations and results of our hybrid algorithm are discussed in Chapter 4. Since the location problem in random deployments has become a challenge for the research community, this motivated us to evaluate the performance of our scheme in two main scenarios: sparse and dense networks. Finally, Chapter 5 gives conclusions of our work and discusses future work on the sensor localization problem. 


\section{Chapter 2}

\section{Related work}

\subsection{Classification of Location Algorithms}

\subsubsection{Centralized and Distributed Algorithms}

One type of classification of the localization algorithms is based on their computational structure. For this category, algorithms can be centralized or distributed. For the first type of algorithms a central node has to be deployed which would be able to perform all the processing (i.e. once it has computed the positions of the nodes, the information is passed back to them). One drawback of this approach is the power consumption that it requires, which is unfeasible for ad-hoc sensor networks [2]. Furthermore, the scalability is compromised affecting the density of the networks. The second type of algorithms are distributed in nature and can be run in parallel performing inter-node communication. The estimation of a node position is achieved by retrieving information from other nodes (its neighbors) in the network. For this reason, distributed algorithms do not need a central node to perform the computations [2].

Two variants exist for distributed algorithms. In the first variant, known as 
beacon-based distributed algorithms, the nodes use a set of anchors in order to obtain range measurements for location purposes $[3,4,7]$. The second variant of algorithms tries to optimize a global metric following a distributed procedure such as $[8,9]$. In certain cases, a process of refinement is implemented on the algorithms in order to minimize the error locally [9]. An overall error function obtained from all the vicinities of the network will define the global metric of performance.

\subsubsection{Single-hop and Multi-hop Algorithms}

\section{Single-hop Algorithms}

In single-hop localization systems, an unknown sensor estimates its position using direct range measurements from the anchors. In the case of two dimensions, the node to be localized should be in range of at least three anchors. The range estimate of a node $i$ to an anchor $j$ denoted as $r_{i j}$ can be expressed in terms of the coordinates of $i$ and $j$ by the following expression,

$$
r_{i j}^{2}=\left(x_{i}-x_{j}\right)^{2}+\left(y_{i}-y_{j}\right)^{2}
$$

which is equivalent to

$$
r_{i j}^{2}=x_{i}^{2}-2 x_{i} x_{j}+x_{j}^{2}+y_{i}^{2}-2 y_{i} y_{j}+y_{j}^{2}
$$

If a node has connectivity with three anchors $\{j, k, h\}$, then three equations can be written for each anchor and the node $i$. It should be noticed that quadratic terms appear in the expressions. To remove such terms, we substract one of the equations from the rest, leaving the two variables of interest (i.e. $x_{i}, y_{i}$ ) in the 
two remaining equations. For instance, assume the following:

$$
\begin{aligned}
& r_{i j}^{2}=x_{i}^{2}-2 x_{i} x_{j}+x_{j}^{2}+y_{i}^{2}-2 y_{i} y_{j}+y_{j}^{2} \\
& r_{i k}^{2}=x_{i}^{2}-2 x_{i} x_{k}+x_{j}^{2}+y_{i}^{2}-2 y_{i} y_{k}+y_{k}^{2} \\
& r_{i h}^{2}=x_{i}^{2}-2 x_{i} x_{h}+x_{j}^{2}+y_{i}^{2}-2 y_{i} y_{h}+y_{h}^{2}
\end{aligned}
$$

Then, following the method above described, we can obtain,

$$
\begin{aligned}
& r_{i j}^{2}-r_{i h}^{2}=x_{i}\left(2 x_{h}-2 x_{j}\right)-y_{i}\left(2 y_{h}-2 y_{j}\right)+y_{j}^{2}-y_{h}^{2} \\
& r_{i k}^{2}-r_{i h}^{2}=x_{i}\left(2 x_{h}-2 x_{k}\right)-y_{i}\left(2 y_{h}-2 y_{k}\right)+y_{k}^{2}-y_{h}^{2}
\end{aligned}
$$

It is clear that this system is solvable in order to find the values of $x_{i}$ and $y_{i}$. The method is known as trilateration. If a system has more than two equations the linear system is resolved using least squares. This process can be found in the literature as multilateration. From the explanation given, it becomes relevant the fact that each unknown sensor do not need to have a global vision of the network. The node estimate can be obtained from the location information provided by the anchors which it has direct communication. Therefore, the method can be executed in a distributed manner. One drawback of this approach is that a node may have several anchors in the vicinity. This task can be tedious if the anchors are placed manually. Furthermore, if a sensor deployment is done randomly, some unknown sensors may be isolated from the anchors and may not retrieve their coordinates in order to estimate their positions. Fig. 2.1 illustrates the single-hop scheme. 


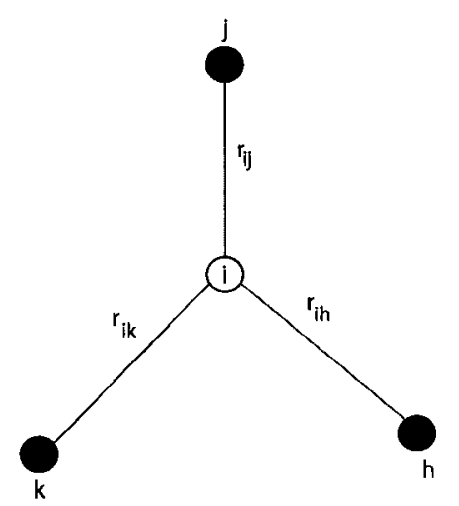

Figure 2.1: Single-hop Localization

\section{Multi-Hop Algorithms}

In contrast to single-hop localization, multi-hop localization is a technique to estimate the positions of the nodes using non-adjacent anchors. Consequently, multi-hop localization can not be reduced to a system of linear equations, as shown in the trilateration and multilateration methods. Additionally, the performance of a multi-hop localization algorithm varies from one topology to another since cells or clusters are not independent and they should work cooperatively. Therefore, it has been challenging to evaluate the performance of the algorithms based on this scheme through physical implementations and the research community has prefered to analyze them on theory and simulations $[6,7,10]$. Fig. 2.2. shows a topology in which an unknown sensor $i$ does not have adjacent anchors and single-hop localization can not be applied. Previous works such as [6,7] have proposed alternatives for this problem using multi-hop localization. 


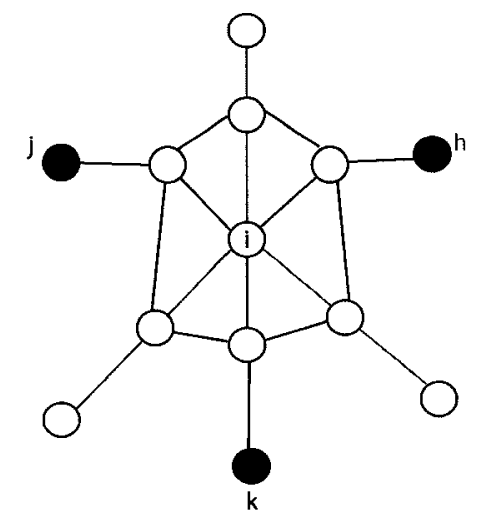

Figure 2.2: Multi-hop Localization

\subsubsection{Range-based and Range-free Algorithms}

\section{Range-based Algorithms}

Range-based schemes rely on computing interdistances in order to infer the nodes estimates. The techniques used for obtaining such range estimates are known as ranging technologies. In certain cases, these estimations require to use extrahardware; however range-based algorithms provide higher accuracy than rangefree schemes. Following, we describe a representative set of ranging technologies.

\section{(1) Received Signal Strength (RSS) and Connectivity}

Range estimates obtained from SS measurements. Due to the advantage that each node has a radio, this can be used to measure the voltage it receives. For location estimation purposes, the research community has observed that the power of the transmitted signal diminishes proportionally with the square of the distance, for non noisy conditions [11]. From this fact, it is possible to compute range estimates in basis of SS measurements [12-15].

The advantage of this approach is that all sensors have radios in order to transmit their data; therefore, no extra-hardware is needed. RSS technol- 
ogy is inexpensive and simple to implement in the nodes. However, the unpredictable behaviour of the signals in noisy environments due to multipath and shadowing, necessitates that other ranging technologies may be used for location estimation purposes.

Generally, a random model is used in order to analyze the radio propagation considering the effects of multipath and shadowing [12]. Multipath can be explained in terms of multiple signals with different amplitudes and phases which arrive to the receiver. Shadowing is attributed to the attenuation of a signal due to obstacles which are present in the environment [11].

Connectivity. Since SS measurements seem to behave randomly and highly dependent of the phenomena of the environment (e.g. fading, shadowing, diffraction), RSS can be used only for obtaining information if two sensors are connected or not. If two sensors are separated less than a maximum reachable radio range, then these two sensors are connected with high probability. Indeed, a yes-no decision is obtained for every pair of sensors.

(2) Time Difference of Arrival (TDoA) A more robust ranging technique than RSS is Time Difference of Arrival. The range estimates between the transmitters and receivers involve the computation of both the time stamped of the radio signal and the time stamped of the acoustic signal. Specifically, in TDoA, a transmitter sends the RF signal. After it has been received by a listening sensor, a time $t_{\text {radio }}$ is stamped. Likewise, when the listening node receives the acoustic pulse, it computes the corresponding $t_{\text {sound. }}$ In general, the transmitter has to wait for a period of $t_{\text {delay }}$, defined by the interval of time for which the transmitter sends the radio and acousting signals. An illustration of the TDoA technique [2] is shown in Fig. 2.3. Once the quantities have been determined, the range estimate 


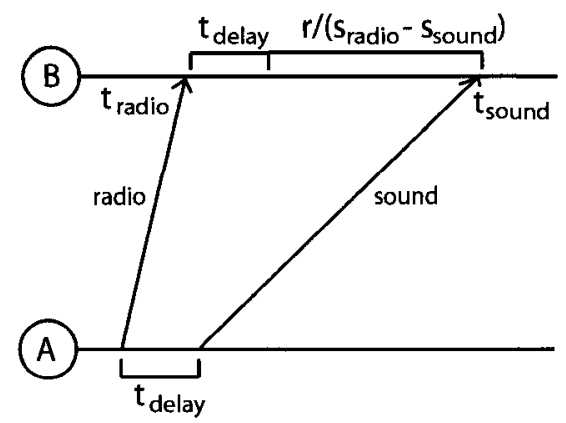

Figure 2.3: TDoA Technique

can be obtained as follows [11],

$$
r=\left(s_{\text {radio }}-s_{\text {sound }}\right)\left(t_{\text {sound }}-t_{\text {radio }}-t_{\text {delay }}\right)
$$

where $s_{\text {radio }}$ is the speed of the radio signal and $s_{\text {sound }}$ is the speed of sound. In contrast to the RSS scheme, TDoA forces to use extra-hardware in the sensors, such as a microphone and a speaker. It has been shown that TDoA has a good performance under LOS (Line of Sight) conditions. Nevertheless, some environments are not suitable for dealing with LOS. The robustness of the TDoA scheme relies on the computation of time differences of the signals instead of the computation of the signal magnitude, which is in general, highly variable.

(3) Angle of Arrival (AoA) Another approach to obtain information useful for location algorithms is by means of angle measurements rather than range estimates. The AoA technique solves the question on how to obtain the direction to neighboring sensors. One method relies on the usage of a sensor array. Therefore, each node is constituted by several microphones in the case of acoustic signals or antennas in the case of RF signals. The AoA can be obtained from the diferrence in arrival times of the signal transmit- 
ted at each of the microphones or antennas in the array. A second method consists on computing the ratio of the SS measurements between two antennas located on the sensor. If the main beams of these sensors overlap, then the AoA can be obtained from the ratio of both SS measurements [2].

\section{Range-free Algorithms}

Range-free algorithms find alternative solutions to those given by range-based schemes for the location problem. $[3,4,6,7]$. These solutions are based on retrieving connectivity information from neighbors around the sensors. The range-free schemes arise due to the unreliability of adopting either an empirical or theorical model to map signal strengths into distance estimates. Furthermore, phenomena

such as multipath, interference, etc., make range measurements innacurate [3]. Following, are some representative algorithms in this second category.

Bulusu et. al [4] proposes a simple approach for the localization problem in sensor networks. In order to estimate the positions of the unknown sensors, the algorithm consists on computing the centroid of the set of audible anchors for each unknown sensor. An anchor becomes audible when it is above of a threshold defined as the ratio between the number of sent and received beacons.

Authors in [6] propose APS (Ad-hoc Positioning System), a distributed algorithm. They observed that the anchors can contact the unknown sensors in a hop by hop manner. Specifically, anchors flood their positions throughout the network counting the number of hops until the message reaches to the target node. Several propagation methods are proposed by authors in [6] in order to infer how far is each unknown sensor from the anchors. Once the unknown sensors have obtained the shortest distance in hops, the algorithm computes the average distance per hop or correction factor. Both quantities are used for the unknown 
sensor in order to compute its distances to the anchors. Once an unknown sensor has obtained such range estimates to at least three anchors, it computes its position using a procedure based on multilateration. Another algorithm known as Amorphous Positioning Algorithm [7] includes a first phase similar to [6]. In their research the authors, they use the hop count as a metric in order to compute inter-node distances. Therefore, a hop count between two sensors defines the length of the shortest path in the graph. Now, consider that the maximum radio range per communication hop is $R$. Supposedly, if two sensors are separated $h$ hops, then the distance between these two sensors, $d$, is less than $R h$. In order to obtain some gain in the accuracy, researchers in [7] suggest to average distances with neighbors. Each sensor recollects the gradient values from the neighbors and itself in order to compute an average. Once a node has obtained the estimated distances to at least three anchors, the multilateration scheme can be used to estimate the node's location.

\subsection{The APIT Algorithm: A range-free scheme}

\subsubsection{Overview}

APIT [3] is a range-free algorithm which requires $N$ nodes acting as anchors for the $M$ unknown sensors. The core of the algorithm consists on defining a set of triangles for each unknown sensor among the audible anchors. Audible anchors are those nodes which can be listened by the unknown sensor. The fact that a particular node resides inside or outside of a triangle would allow to define the region in which we may locate the node. Intuitively, by increasing the number of triangles in which the node possibly resides, we are reducing the resulting region in order to provide a good location estimate. 


\subsubsection{Steps for APIT}

The following steps describe how to estimate the position of an unknown sensor $a$ through APIT [3].

Generation of triangles: An unknown sensor $a$ will perform k-combinations, where $k=3$, from the set of $n$ audible anchors in order to define its set of triangles. For instance, if an unknown sensor has 6 audible anchors, then the number of triangles generated are $\left(\begin{array}{l}6 \\ 3\end{array}\right)=20$.

Acquisition of beacon information: In this stage, node $a$ receives beacons from the audible anchors $A, B, C$ containing the IDs and positions of the anchors. It also receives the signal strengths of $A, B, C$ (i.e. $S S_{a A}, S S_{a B}, S S_{a C}$ ). Likewise, neighbors will receive the signal strengths of the anchors and they beacon this information to $a$. For instance, if node $x$ is neighbor of node $a$, then $a$ will receive (i.e. $S S_{x A}, S S_{x B}, S S_{x C}$ ). Note that each neighbor should have nodes $A, B, C$ as audible anchors, in order to have a merged table in $a$.

APIT test: The APIT test which is formulated by authors in [3] claims the following situation in order to decide if a node is inside or outside of a triangle: "If no neighbor of node $a$ is further from/closer to all three anchors $A, B, C$ simultaneously, a assumes that it is inside triangle ABC. Otherwise, a assumes it resides outside this triangle."

The manner in which the APIT test runs in the APIT algorithm is comparing the set of signal strength measurements of each neighbor $\left(S S_{x A}, S S_{x B}, S S_{x C}\right)$ and the set of signal strength measurements of the unknown sensor $a\left(S S_{a A}, S S_{a B}, S S_{a C}\right)$. If one neighbor has a set of SS measurements smaller or greater than the set of SS measurements of $a$, then $a$ is outside of the triangle. Fig. 2.4 illustrates the collection of beacon information so the APIT test can be run. The unknown sen- 


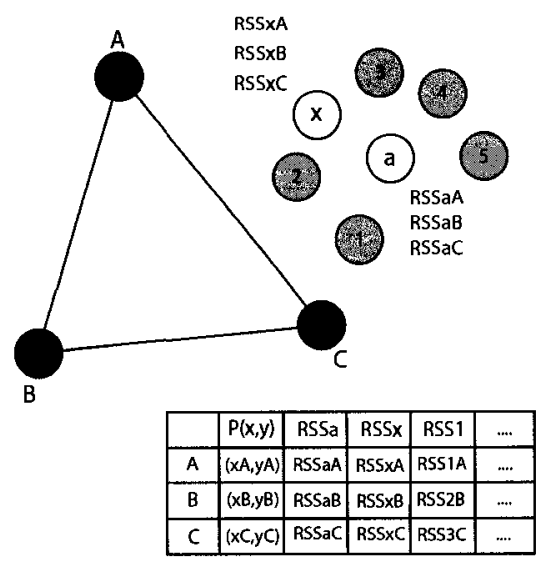

Figure 2.4: The APIT test.

sor $a$ receives the signal strengths of anchors A,B and C. Neighbor $x$ recollects this information as well. A merged table in node $a$ contains its own set of SS measurements and the neighbors sets of SS measurements. The APIT test is run comparing the set of SS measurements of $a$ and the set of SS measurements of its neighbors.

APIT Aggregation: So far we know if node $a$ has decided if it is inside or outside of the triangle. Possibly, there is more than one triangle generated from the combination of all the audible anchors of node $a$. If each triangle generates an inside or outside decision, then we can aggregate the individual results. This is achieved through a grid array which defines smaller regions. For each inside decision the grid area is increased, otherwise it is decreased. The step is shown in Fig. 2.5.

Position estimation: Once we have identified the region with the maximum counter after increasing-decreasing the grid areas for node $a$, we can compute the COG (Center of Gravity) for this region. This will constitute the final estimate for node $a$. 


\begin{tabular}{|c|c|c|c|c|c|c|c|}
\hline $\mathrm{A}$ & 1 & 0 & 0 & 0 & 0 & 0 & 0 \\
\hline 0 & 1 & 1 & & 1 & 0 & 0 & 0 \\
\hline 0 & 1 & 1 & 1 & 1 & 1 & & 0 \\
\hline 0 & 0 & 2 & 2 & 2 & 2 & -1 & 0 \\
\hline 0 & 2 & 2 & 0 & 2 & 2 & -5 & $F^{-1}$ \\
\hline 0 & 0 & 2 & 2 & 2 & 1 & -1 & -1 \\
\hline 0 & 0 & 1 & 1 & 1 & & 0 & 0 \\
\hline 0 & 0 & 0 & 0 & 0 & 0 & 0 & 0 \\
\hline 0 & 0 & 0 & 0 & 0 & 0 & 0 & 0 \\
\hline
\end{tabular}

Figure 2.5: APIT Aggregation. For each inside decision the counter is increased, otherwise it is decreased. Three triangles are shown, where two of them made inside decisions and the other one made an outside decision

\subsubsection{The parameter DOI and the radio model used for APIT}

In this section we briefly describe the parameter DOI (Degree of Irregularity) [16] which is used in the APIT algorithm [3] in order to simulate the effect of noise on the radio signals emmited by the sensors. DOI denotes the irregularity of the radio pattern and it is defined by its authors as [16]: "the maximum radio range variation per unit degree change in the direction of radio propagation". If DOI increases, the radio pattern will show more irregularities. Likewise, if DOI is close to zero, the radio pattern will tend to an isotropic one. That is, the variations of the signal would depend mainly with the distance and not with the direction. The parameter DOI is used to denote the anisotropic property of RIM (Radio Irregularity Model). RIM is a model in which radio variations are affected by experimental data (DOI values). In this model, the received signal strength is represented as follows [16],

$$
R S S=P_{t}-\text { Pathloss } * K_{i}+\text { Fading }
$$


where $R S S$ is the received signal strength in $\mathrm{dBm}, P_{t}$ is the power transmitted and $K_{i}$ is a coefficient that adjusts the path loss in a given direction $i,(0 \leq i \leq 360)$. $K_{i}$ is defined as,

$$
K_{i}= \begin{cases}1 & i=0 \\ K_{i-1} \pm \text { Rand } * D O I & i \in N \\ K_{s}+(i-s) *\left(K_{t}-K_{s}\right) & i \notin N\end{cases}
$$

where $N$ is the set of natural numbers, Rand is a random number that follows the Weibull distribution [16], $s=\lfloor i\rfloor$, and $t=\lceil i\rceil$.

The goal of RIM [16] is to cover the gap between the isotropic model and the real radio model, the latter studied through physical experimentation $[17,18]$. Likewise, RIM gives an approach for studying the variation of signal strengths considering both distance and direction of propagation. Therefore, other models used in $[12,13]$ do not hold well for different noisy conditions. Furthermore, the accuracy of RIM has been verified in real environments [16] through several experiments.

Two radio patterns are shown in Fig. 2.6. Meanwhile 2.6(a) does not show any radio irregularity (i.e. perfect communication range), 2.6(b) denotes how the irregularity becomes present when DOI is different to zero. 


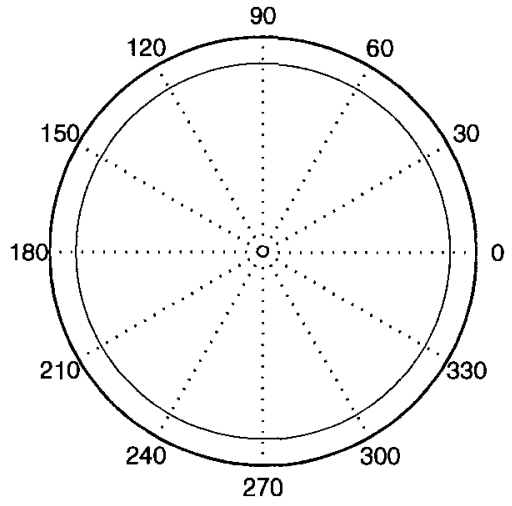

(a) $\mathrm{DOI}=0$

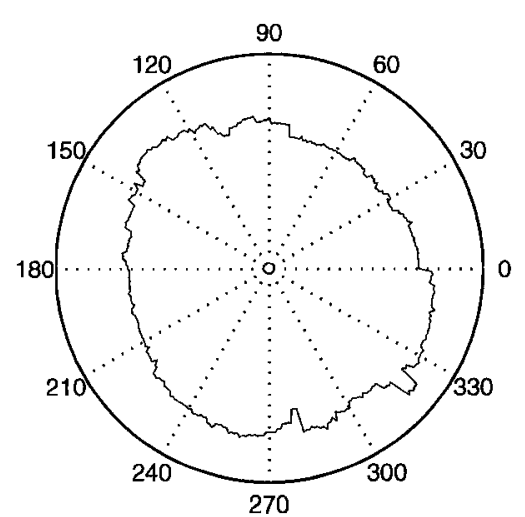

(b) $\mathrm{DOI}=0.05$

Figure 2.6: The DOI parameter. Case (a) shows a perfect circular radio model due to the abscense of noise. Case (b) denotes range variations due to noise. 


\section{Chapter 3}

\section{A hybrid scheme}

\subsection{Drawbacks of the APIT algorithm}

In order to introduce how the performance of the APIT algorithm can be improved, it is important to ennumerate some of its drawbacks.

APIT test: From the description of the APIT test we should observe that each inside-outside decision, depends on the size of the neighborhood and the llocation of the nodes in the vicinity in order to make an accurate decision. The authors in [3] observed this situation and claimed that a node density (ie. the average number of nodes per node radio range) above 6 allows a good performance of the algorithm. Refer to Fig. 3.1 for the condition of connectivity required by the APIT test.

However, an interesting approach would be to propose a test in which a target node would not rely on the information provided from the neighborhood in order to perform an inside-outside decision. In the beacon exchange phase, the target node collects the position, ID, its own set of SS measurements and the

signal levels of their neighbors obtained from the anchors. Probably, a subset 


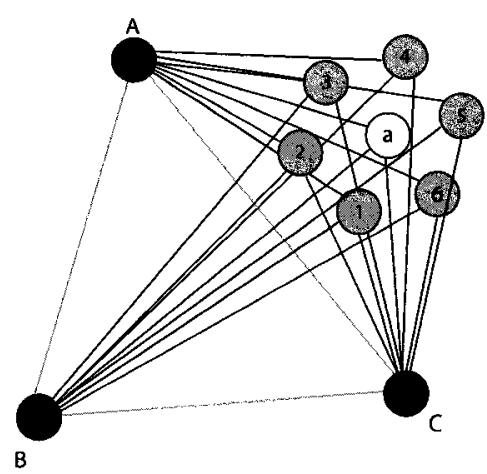

Figure 3.1: Drawback on APIT test: Strong condition of connectivity between unknown sensor-anchors and neighborhood-anchors

from the neighborhood would be in range of the anchors and probably another subset will not. As a result, it is required that the target node and a considerable number of neighbors should be in range with the anchors. Obviously, we can not guarantee a complete connectivity between unknown sensor-anchors and neighborhood-anchors and this is certainly a disadvantage especially in sparse sensor networks.

Number of localized nodes: Authors in [5] observed that the APIT algorithm has the drawback of not determining the positions of all nodes. The first reason is that a node can not determine its own position because it does not have enough audible anchors (at least three) in order to run the APIT test. Fig. 3.2 shows this case.

A second reason is that a node may not fall inside any triangle from the set of combinations performed among the audible anchors. Computing the position of the node in this last situation may not be possible. Likewise, the estimation of its position would contribute to increase the error of the entire network. An approach found in [5] refers to this drawback, but the authors prefered to propose a different algorithm instead of improving APIT.

Accuracy in the estimation: Since APIT belongs to the category of range-free 


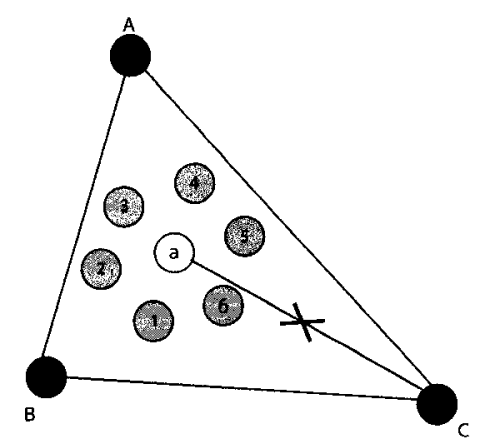

Figure 3.2: Anchor $\mathrm{C}$ is not audible by the unknown sensor $a$ whose position can not be estimated

schemes, it provides rough estimates. Alternative schemes such as DV-Hop [6] employ an iterative refinement phase that helps to approximate a set of initial estimates to the positions that best fit with the measured distances.

In the following section we describe in detail possible solutions for the disadvantages above explained.

\subsection{The enhanced APIT algorithm}

\subsubsection{An Alternative Test: RF profiling}

We consider a case where there is a triangle (defined by anchors $A, B, C$ ) and a sensor $a$ for which the position where it resides (inside or outside the triangle) is unknown. The APIT test proposed in [3] gives an approach for this situation; however, we wish to investigate an alternative method. Assume that we use a grid array that divides the area of interest in equal regions. For each point of the grid, triplets of SS measurements can be recorded. This process is known as $R F$ profiling [19]. For instance, Point 3 in Fig. 3.3 has a triplet of SS measurements $\left(S S_{3 A}, S S_{3 B}, S S_{3 C}\right)$. We denote this triplet as the set of recorded measurements. Sets of triplets will constitute a database of recorded SS measurements. An un- 
known sensor $a$ will perform signal strengths measurements from the anchors $A, B, C\left(S S_{a A}, S S_{a B}, S S_{a C}\right)$. We refer to this triplet as the set of observed measurements. In a subsequent stage, error values are computed between each triplet of recorded measurements and the triplet of observed measurements. The minimum of all these errors, will identify the triplet of recorded measurements which is closest to the set of observed measurements. For illustration purposes, consider the set of recorded SS measurements of Point $3\left(S S_{3 A}, S S_{3 B}, S S_{3 C}\right)$ and the set of observed SS measurements of $a\left(S S_{a A}, S S_{a B}, S S_{a C}\right)$. The distance in signal space or error can be measured as,

$$
\sqrt{\left(S S_{a A}-S S_{3 A}\right)^{2}+\left(S S_{a B}-S S_{3 B}\right)^{2}+\left(S S_{a C}-S S_{3 C}\right)^{2}}
$$

The grid point that belongs to the identified set of recorded measurements will be a good approximation to determine if $a$ is inside or outside the triangle.

Such technique was used in [19] in order to estimate the location of a user; however, RF profiling can be a model for an alternative test. Furthermore, we use the RIM Model [16] as our propagation model in order to generate signal strengths since it is suitable for sensors. It is important to mention that the authors in [16] used another propagation model appropiate for high-power devices and indoor environments.

We generate the set of recorded measurements using (2.4). For simplicity, we consider that the $S S$ measurements decay logaritmically over distance due to the effect of Pathloss and there is no Fading in our system.

Using (2.4) and knowing the distances from each grid point to the anchors $A, B, C$, we can generate triplets of $S S$ measurements. This avoids the tedious task of gathering data using an empirical method as the one shown in [19]. It also 


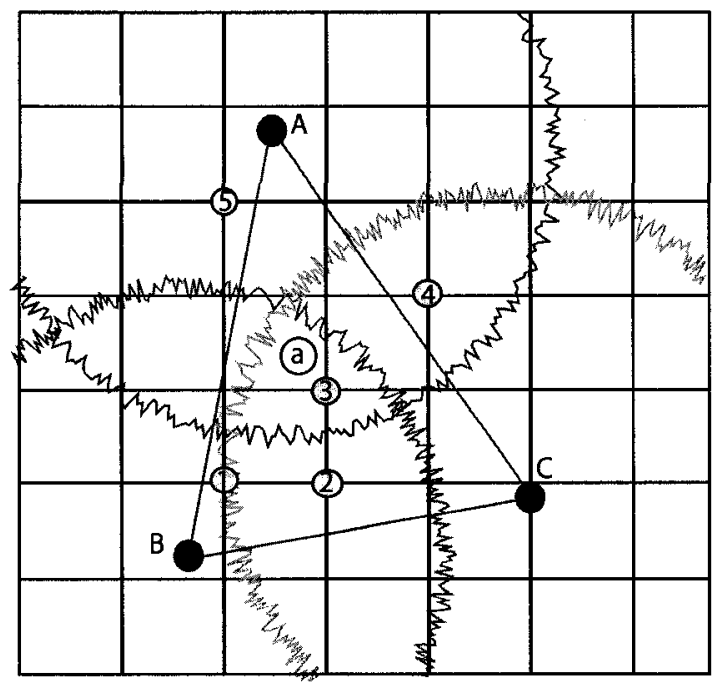

Figure 3.3: An Alternative Test. Triangle with three anchors A,B,C as verteces. Each irregular radio pattern is illustrated around each anchor. In order to decide if $a$ is inside or outside of the triangle, a set of recorded SS measurements and a set of observed measurements of $a$ to A,B,C are needed. Points 1,2,3 and 4 are some points from the grid in which we can record triplets of SS.

emulates the process of recording $S S$ measurements as if the unknown sensor $a$ would be allocated in different positions of the grid.

Hereinafter, we denote the $R F$ profiling process above described as the alternative test.

\subsubsection{Iterative APIT algorithm}

For this case, we run the APIT algorithm in order to estimate the positions of some unknown sensors. Once we have estimated such positions, we are able to use this data to estimate the positions of additional unknown sensors not located by the first run of APIT. Indeed, both the first set of estimates and the anchors can be used as a new set of anchors in order to compute the positions of another set of nodes. However, this approach may cause the location error to propagate from one iteration to another. This can be attributed to the fact that 


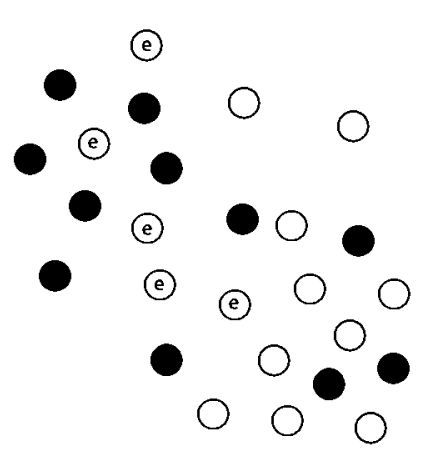

(a) APIT is run

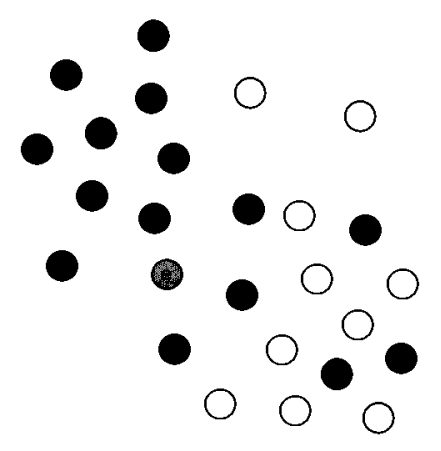

(b) New anchors are assigned

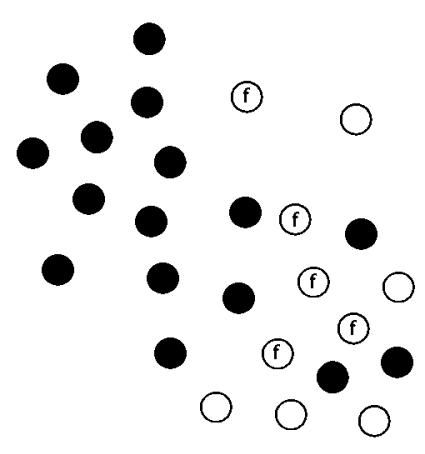

(c) Iterative APIT finishes

Figure 3.4: Iterative APIT. In Case (a), the positions of the unknown sensors are estimated through APIT using the anchors (black nodes). In Case (b), the first set of estimates (gray nodes) and the anchors are used as a new set of anchors. In Case (c), a second set of estimates is obtained (nodes denoted with letter $f$ )

the estimates that are used as new anchors may not match their true positions accurately. A solution for this situation is provided by the Refinement procedure that we propose later.

Fig. 3.4 illustrates the manner in which the APIT algorithm is executed iteratively.

Two advantages are obtained using Iterative APIT. First, Iterative APIT estimates more nodes positions than APIT using the same number of anchors.

Second, the efficiency of the algorithm will not be significantly reduced because the first set of estimates and the anchors are going to be used to build more triangles. Note that the success of the algorithm relies on building more regions; therefore reducing the area in which the nodes can lie in.

As an illustration, consider Fig. 3.5. It can be observed that the first set of estimates and the anchors can be used together to generate more triangles and, possibly, estimate the positions of the three nodes denoted with letter $f$. As a remark, it is worth mentioning the following. We run the APIT algorithm twice. Another possibility would be to run the APIT algorithm more than two 


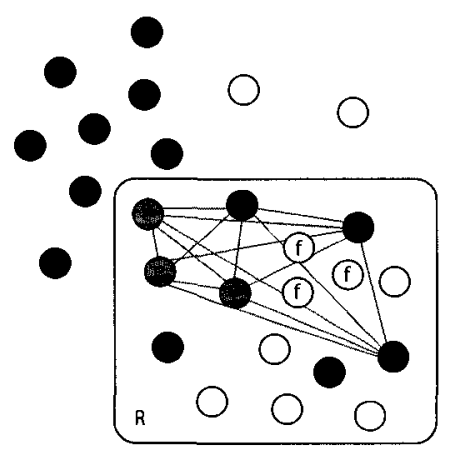

(a) Network in which Iterative APIT is run

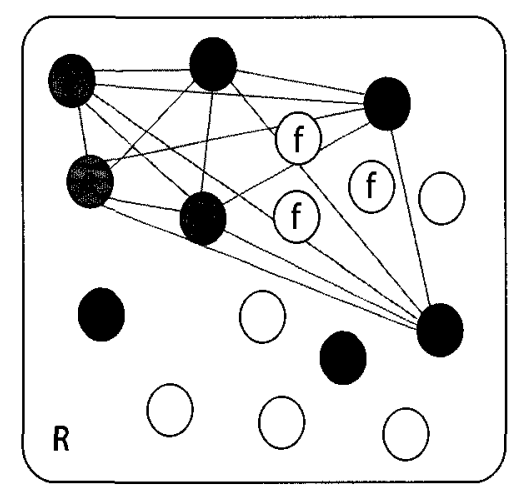

(b) Subnetwork R

Figure 3.5: Triangulation in Iterative APIT. A subnetwork $R$ is shown illustrating a set of anchors (black nodes), the first set of estimates (nodes denoted with letter $e$ ), and the second set of estimates (nodes denoted with letter $f$ )

times. However, the computational complexity would increase since more nodes estimates would be acting as anchors and more exchanges of beacons information would be necessary.

Furthermore the Iterative APIT can be applied also in a different variant. This consists on the following steps.

The first step is to try to estimate the position of an unknown sensor through APIT. If it succeeds, then the node is used as a new anchor. Secondly, the new anchor (if it exists) and the anchors used to estimate its position are used to estimate the position of a second unknown sensor. This procedure is repeated continuously. That is to say, we go to the first step and repeat the cycle. This second variant of Iterative APIT finishes until we are not able to estimate more node positions.

As a quick example consider Fig. 3.6. $\{A, B, C\}$ are audible anchors for the unknown sensor $a_{t}$. The APIT algorithm is run and a position $a$ is estimated for such sensor. $a$ is assigned as a new anchor. $\{a, A, B, C\}$ are audible anchors for the unknown sensor $b_{t}$. The APIT algorithm is run considering all the combi- 


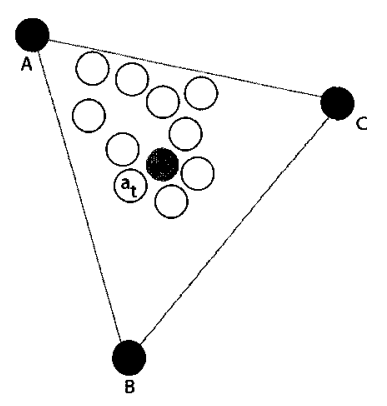

(a) APIT is run

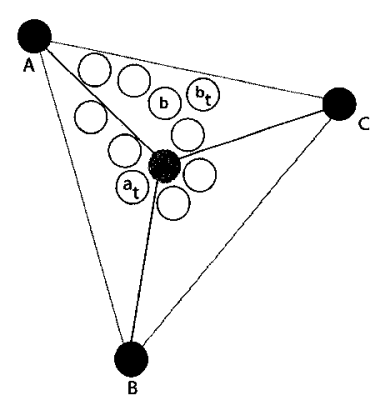

(b) New anchor is assigned

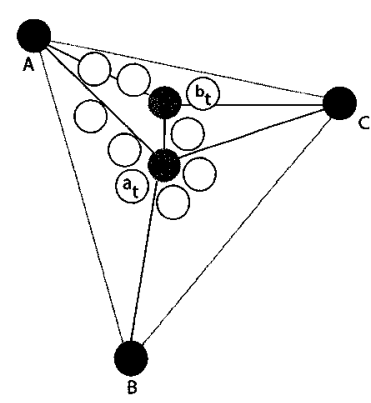

(c) Estimation of another unknown sensor

Figure 3.6: A second variant of Iterative APIT

nation of triangles $(A B C, a B C, a A C, a A B)$ and a position $b$ is estimated for such sensor. $b$ is assigned as a new anchor and the procedure continues.

The estimation of the nodes positions through this variant of Iterative APIT is performed sequentially. A drawback of this algorithm is that the algorithm has to wait for a previous estimation of a node position in order to perform the subsequent estimation. We did not evaluate this approach in our experiments and we left it as future work.

\subsubsection{Refinement for APIT algorithm}

The refinement procedure is based on the multilateration scheme $[2,6,7,20]$. Having an initial estimate of the unknown sensor $s_{o}$ obtained through APIT, and knowing the position of an anchor $b$, we can define the estimated distance as $\left\|s_{o}-b\right\|$. Likewise, the method uses the observed ranges $r_{i}$ which can be obtained from SS measurements. Additionally, a target node may have $m$ audible anchors $(m \geq 3)$.

It is clear that we are adopting a range-based scheme at this point. The purpose is to improve the accuracy of the estimates obtained from APIT. These estimates are close to the true positions in certain conditions [3]; however, the multilateration 
procedure will refine even more such estimates. Multilateration will converge when a method can provide good approximations to the true positions.

The goal of the multilateration scheme [2] is to minimize the error between the estimated and observed ranges, in order to find $s$ (i.e. the position of the unknown sensor).

The expressions found in [2] give an approach to find an estimate of $s$ using multilateration, minimizing an error function $E(s)$ in each step of the iterative process.

$$
s=\operatorname{argmin} E(s)=\sum_{i=1}^{m}\left(\left\|s-b_{i}\right\|-r_{i}\right)^{2}
$$

If we define $e\left(s, b_{i}\right)=\left(\left\|s-b_{i}\right\|-r_{i}\right)$, and we approximate this error function with a first order Taylor series around the initial estimate $s_{o}$, the following can be stated:

$$
\begin{array}{r}
e\left(s, b_{i}\right) \approx e\left(s_{o}, b_{i}\right)+\nabla e\left(s_{o}, b_{i}\right)\left(s-s_{o}\right) \\
=\nabla e\left(s_{o}, b_{i}\right) s-\left(-e\left(s_{o}, b_{i}\right)+\nabla e\left(s_{o}, b_{i}\right) s_{o}\right) \\
\nabla e\left(s, b_{i}\right)=\frac{s-b_{i}}{\left\|s-b_{i}\right\|}
\end{array}
$$

Now, we can rewrite (3.1) as,

$$
s \approx \operatorname{argmin} \sum_{i=1}^{m}\left(\nabla e\left(s_{o}, b_{i}\right) s-\left(-e\left(s_{o}, b_{i}\right)+\nabla e\left(s_{o}, b_{i}\right) s_{o}\right)\right)^{2}
$$

The above equation can be expressed in a matrix form as,

$$
s \approx \operatorname{argmin}\|A s-b\|^{2}
$$




$$
A=\left[\begin{array}{c}
\nabla e\left(s_{o}, b_{1}\right) \\
\nabla e\left(s_{o}, b_{2}\right) \\
\cdot \\
\cdot \\
\cdot \\
\nabla e\left(s_{o}, b_{3}\right)
\end{array}\right]
$$

The multilateration scheme [2] can be summarized as follows: Consider a position $s_{o}$ as an initial seed for the optimization. In our case, we choose $s_{o}$ as the initial estimate obtained from APIT, which is a good approximation to the true position. Afterwards, we compute $A$ and $b$ using Eqs. 3.3 and 3.4. These two matrices are used for obtaining a new estimate $s_{o}$ through a least squares minimization.

$$
s_{o}^{\prime}=\left(A^{T} A\right)^{-1} A^{T} b
$$

Then, we are able to compute the difference between error functions $E\left(s_{o}\right)$ and $E\left(s_{o}^{\prime}\right)$. If the difference is less than a predefined threshold $\epsilon$ then $s_{o}^{\prime}$ is the solution. If not, we should recompute $A$ and $b$ and go through the procedure once again. As it can be observed, the process is performed iteratively. 


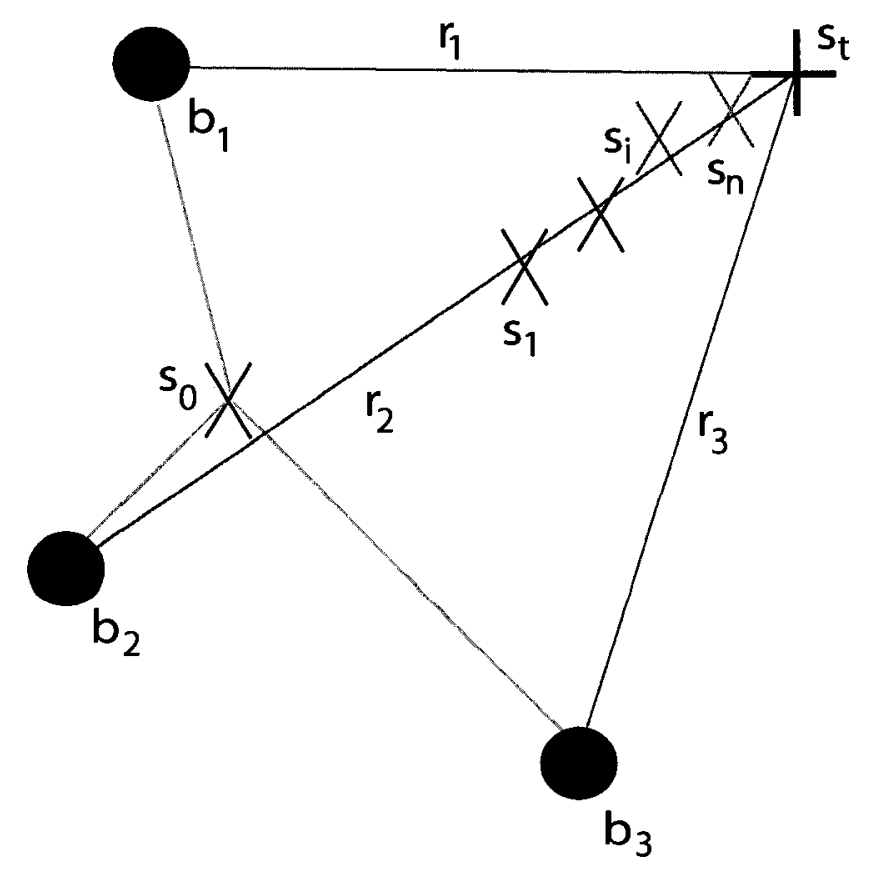

Figure 3.7: Refinement procedure

Note that in the case of heavy-noisy observed ranges, we may avoid to use them in this process. For this situation, it would be preferable to consider the estimates obtained from APIT as our closest approximations to the true positions. It should be remarked that a reduced number of iterations is necessary so that the computational complexity should not be increased in a way that it would exhaust the resources of the sensor network (such as power processing). Therefore, the selection of $\epsilon$ should be a balance between computation and accuracy.

Fig. 3.7 depicts the refinement procedure. $s_{o}$ is the initial estimate obtained from APIT, $\left\{b_{1}, b_{2}, b_{3}\right\}$ are three audible anchors and $s_{t}$ is the true position of the unknown sensor. For each iteration $i$, we want to minimize the difference between the observed measurements $\left\{r_{1}, r_{2}, r_{3}\right\}$ and the estimated measurements $\left\{\left\|s_{i}-b_{1}\right\|,\left\|s_{i}-b_{2}\right\|,\left\|s_{i}-b_{3}\right\|\right\}$. After $n$ iterations, $s_{n}$ is the final position for the unknown sensor. 


\section{Chapter 4}

\section{Simulation and Performance}

\section{Analysis}

\subsection{Simulation Objectives}

Our main goal is to evaluate how each of the enhancements described in Chapter 3 contribute towards improving the performance of APIT. The performance of our scheme and APIT can be evaluated in terms of location error, number of located nodes, and proportion of accurate decisions. The proportion of accurate decisions is obtained from running the APIT test and the alternative test.

\subsection{Simulation Environment and Implementa- tion}

The implementation of our enhanced algorithm has been done using MATLAB, Octave and Shell scripting. Some of our simulations were run using Sharcnet (Shared Hierarchical Academic Research Computing Network), which is a net- 
work that includes clusters of high-performance computers [21] available to the research community.

Implementing our approach required to perform the following tasks.

1. To develop the code for APIT [3].

(a) Implementation of the RIM Model as described in Section 2.2.3.

2. To write the modules for the enhanced algorithm (i.e. alternative test, refinement procedure and Iterative APIT)

(a) Design a routine for our alternative test.

(b) Design a routine for the refinement procedure.

(c) Design a routine for running APIT iteratively.

(c.1) Design a subroutine to assign the new set of anchors (Section $3.2 \cdot 2)$.

APIT and our enhanced algorithm have the feature of building tables and matrices for the APIT Test and the alternative test. This motivated us to select a platform with the efficiency of manipulating them. MATLAB and Octave provided this facility. MATLAB was used to evaluate the performance of the alternative test. Likewise, we selected MATLAB to run our simulations in sparse networks. On the other hand, Octave and Sharcnet were used to run our simulations in dense networks.

We modified the code written in MATLAB in such a way that it could be run in Octave. We performed this task since Octave was already installed on some Sharcnet UNIX clusters. Furthermore, Octave is very similar to MATLAB in terms of functionality.

Our enhanced algorithm is a distributed algorithm. Consequently, we estimate 
the positions of the unknown sensors in parallel. Sharcnet provided us the facility to execute simultaneously the procedures described in Section 2.2.2. Once we have computed the location error of the system and the number of nodes with estimated location for one iteration, we were able to assign the new set of anchors using the methodology described in Section 3.2.2. The second iteration is also executed in parallel. Afterwards, we obtain the location error of the system and the number of nodes with estimated location for the second iteration. Finally, the location error for the total number of nodes estimated is computed.

\subsection{The General Simulation Setup}

In order to pursue our goals described in Section 4.1, we have to define the simulation scenarios and corresponding parameters.

Previous works have studied their algorithms in either sparse (small-scale) [9] or dense (medium-scale) $[6,7]$ networks. This motivated us to analyze the performance of our algorithm on these simulation scenarios. We evaluated the degree of density of a network in terms of the number of nodes deployed in a square field of $10 x 10\left[m^{2}\right]$. For purposes of our research, we consider a sparse network if the density is less than $1 \frac{\text { node }}{m^{2}}$. On the other hand, if it is greater than this value, the network is dense.

Our analysis covers non noisy $(\mathrm{DOI}=0)$ and noisy $(\mathrm{DOI}>0)$ environments. We distribute the sensors randomly over the terrain. We denote $R$ as the maximum radio range for which two sensors can be connected, $N$ as the number of anchors and $M$ as the number of unknown sensors. All nodes (i.e. $N+M$ ) have the same capabilities in terms of power and energy consumption. Furthermore, the $N+M$ sensors have the same radio range $R$. The DOI values used in our experiments 
are within the range of $\left[\begin{array}{ll}0 & 0.7\end{array}\right]$. Although experiments performed in real sensor devices [16] showed that DOI values are small (less than 0.1), we may want to cover a wider range of DOI values. Therefore, we consider for our simulations similar noisy conditions as shown in [3]. Furthermore, this allows us to evaluate the performance of the algorithm in different noisy environments.

Finally, in all our graphs, each data point represents the average value of 10 trials with different random seeds.

\subsubsection{Location Error Metric}

In order to evaluate the performance of our algorithm in terms of location error, we adopted the RMSE (Root Mean Square Error) as our metric. RMSE is defined as,

$$
R M S E=\sqrt{\sum_{i=1}^{M_{e}} \frac{\left(x_{t}-x_{e}\right)^{2}+\left(y_{t}-y_{e}\right)^{2}}{M_{e}}}
$$

where $M_{e}$ is the number of nodes with estimated location, $\left(x_{t}, y_{t}\right)$ are the coordinates of the true positions and $\left(x_{e}, y_{e}\right)$ are the coordinates of the estimates.

\subsection{Performance in sparse networks}

\subsubsection{Simulation Setup for sparse networks}

\section{Placement Model}

We assume small values for $M$ and $N$ since our evaluations are based on sparse networks. The location of the $M+N$ sensors follows a uniform distribution. 


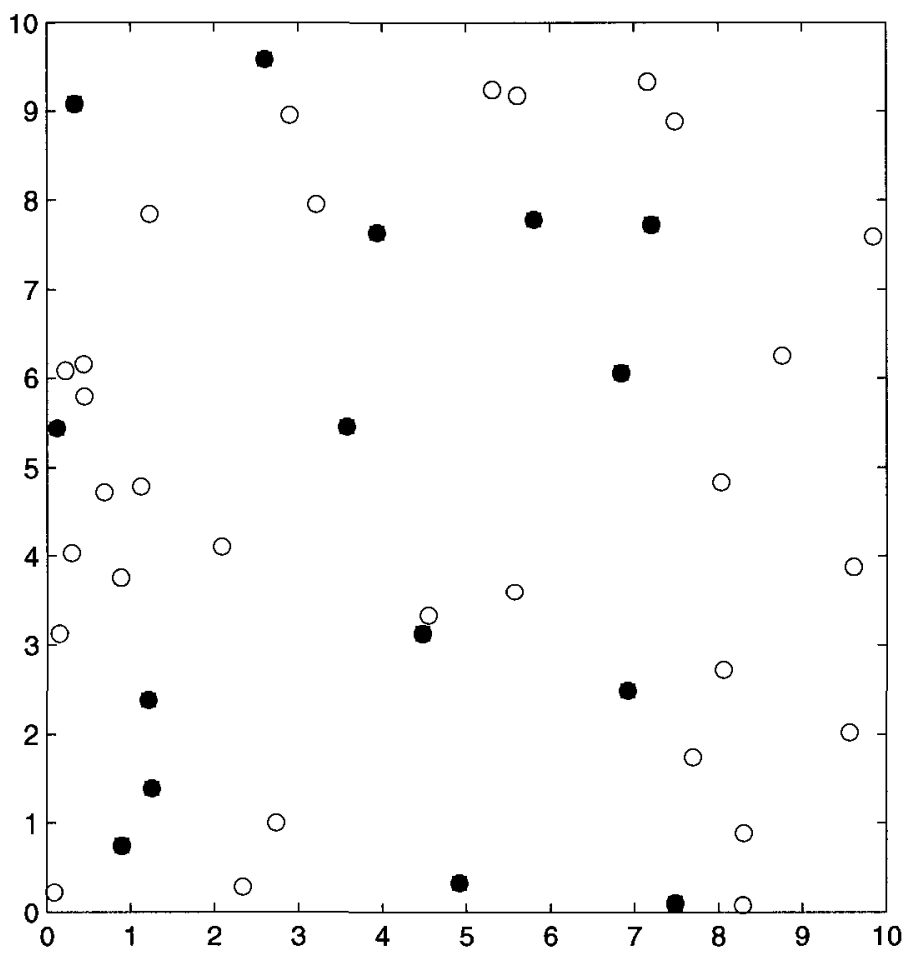

Figure 4.1: Sparse Network. Nodes denoted as (ם) are anchors, nodes shown as (o) are unknown sensors.

\section{Non-noisy and noisy environments}

For both non noisy and noisy environments, the radio range $R$ is set to $4.57[\mathrm{~m}]$. A previous work [17] considered such $R$ value in real experiments. The length of the grid side is set to $0.5[\mathrm{~m}]$ for the APIT Aggregation Step described in Section 2.2.2. Fig. 4.1 depicts a sparse network. 


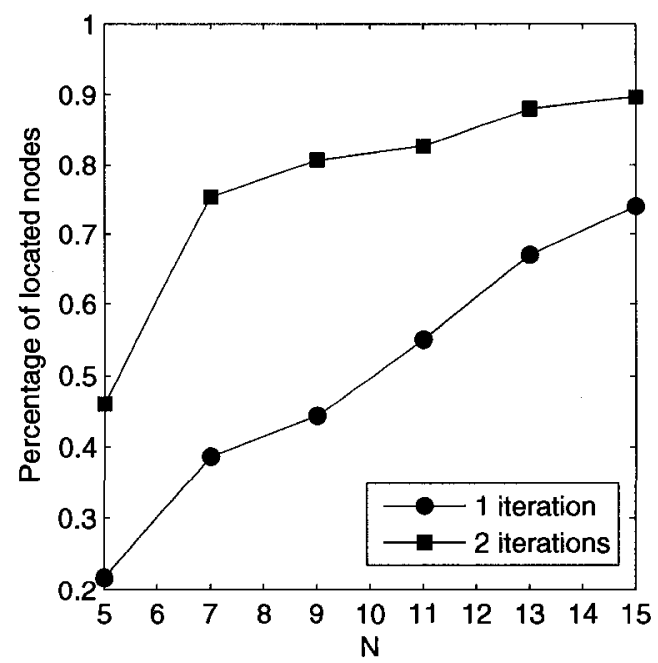

Figure 4.2: Comparison of the number of located nodes when $(-\bullet-)$ APIT is run only once and $\left(-\boldsymbol{\square}_{-}\right)$APIT is run with two iterations. [DOI=0].

\subsubsection{Performance of the enhanced algorithm in non noisy environments for sparse networks}

\section{Determination of nodes using Iterative APIT}

For the experiments in which we study the iterative and refinement procedures, we consider $M=30$ and $N$ varies from 5 to 15 .

In a first experiment, it is of our interest to determine the number of nodes that are estimated when the simple APIT algorithm is run. We compare these results with those that are obtained when APIT is run iteratively. For simplicity, we run APIT only twice. The nodes estimated in the first round, along with the first set of anchors, act as a new set of anchors for the second round in order to estimate a second set of nodes. The results are shown in Fig. 4.2. It can be observed that a greater number of nodes can be estimated when the APIT algorithm is run iteratively. 


\section{Influence of Refinement on APIT}

A second step to observe is how the RMSE is affected by the iterative process. We compare the results obtained from the Iterative APIT with those recollected after running APIT only once. Fig. 4.3 shows the normalized RMSE for different values of $N$. Note that the error values obtained with the estimates of the two iterations are greater than those obtained with the estimates of the first iteration. To refine the positions of the nodes, we applied the process of multilateration as described in Section IV. From Fig. 4.3 we can notice how the accuracy improves when APIT is run only once and the refinement procedure is applied. Intuitively, one may think that the second set of nodes estimates introduces more error than the first set of nodes estimates. The fact that we are using the first set of nodes estimates as anchors for estimating the positions of the remaining nodes can cause the RMSE to increase. Since these anchors are estimates, it may be possible that the error propagates to the second set of anchors. However, the error does not increase significantly (Fig. 4.3) and more nodes are localized (Fig. 4.2). The error propagation is investigated through the analysis described below.

\section{Refinement as an intermediate step for Iterative APIT}

Fig. 4.4 shows the case when APIT is run iteratively without using a refinement process. Three curves are illustrated. Two of them denote the error introduced by each iteration while the other denotes the error introduced by all the estimates obtained from the two iterations.

After observing the benefits of the multilateration process, one may think that we can refine the positions of the first set of nodes estimates before using them as anchors and computing the locations of the remaining nodes. Fig. 4.5 shows this approach. As expected, the error decreases when the multilateration process is 


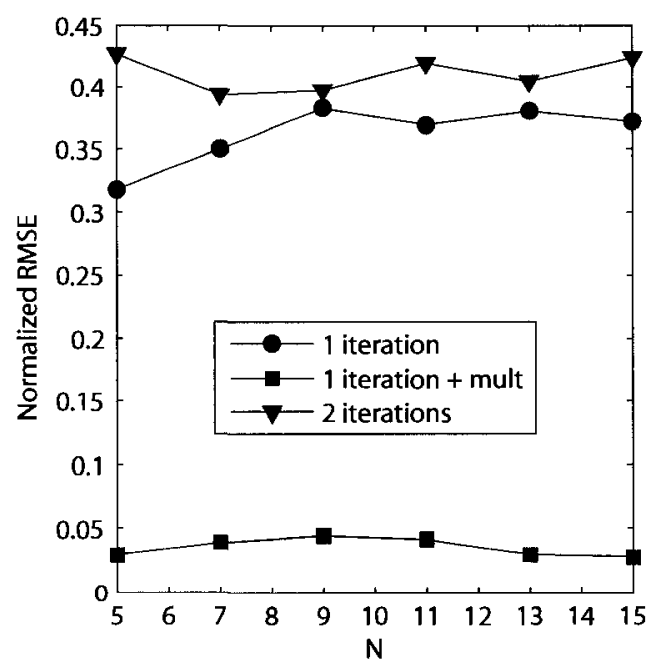

Figure 4.3: Normalized RMSE for three cases: (- - ) RMSE computed with the estimates obtained in one iteration, $(-\mathbf{-}-)$ RMSE computed with the refined estimates of the 1st iteration, (- -$)$ RMSE computed with the refined estimates and estimates of the 2 nd iteration. [DOI $=0]$.

run for the first set of estimates. However, the error is still significant when APIT is run for the second time in order to obtain the second set of estimates [Fig. 4.5 $(-\nabla-)]$. Further research is needed in order to get more conclusions about this behavior. Nevertheless, we should observe that the error for the Iterative APIT using multilateration as intermediate step [Fig. $4.5(-\bullet-)$ ] is less than the error for Iterative APIT without using multilateration [Fig. $4.4(-\bullet-)]$ for all values of $N$, due to the refinement process applied in the first iteration.

\section{Refinement as a final step for Iterative APIT}

In this section we study how the multilateration scheme affects on the estimates after the Iterative APIT has been run. This idea is depicted in Fig. 4.6. For comparison purposes, we also illustrate the case when no multilateration scheme is applied. The benefit of running the multilateration scheme is obvious.

The refinement scheme for Iterative APIT can be applied in two different cases. 


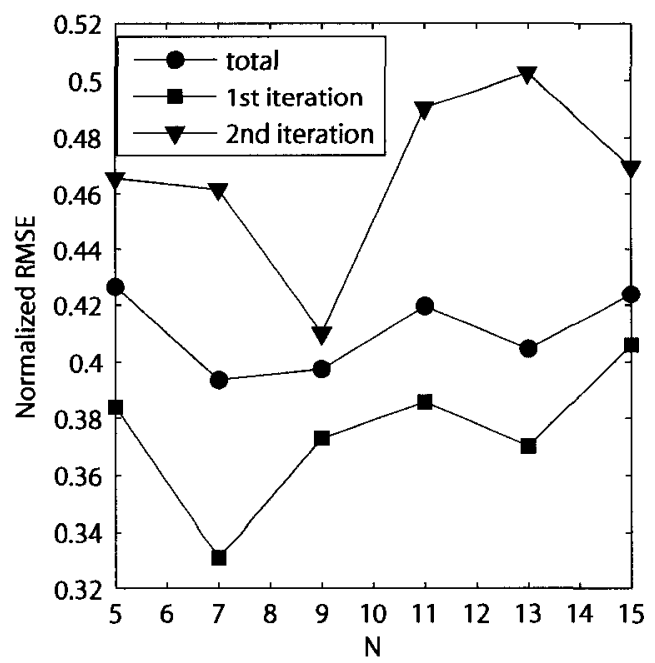

Figure 4.4: Normalized RMSE obtained after APIT is run with two iterations. No multilateration scheme is applied. (-•-) RMSE computed with the estimates obtained in two iterations, (- - ) RMSE computed with the estimates obtained in the 1st iteration, (- -$)$ RMSE computed with the estimates obtained in the 2nd iteration. [DOI $=0]$.

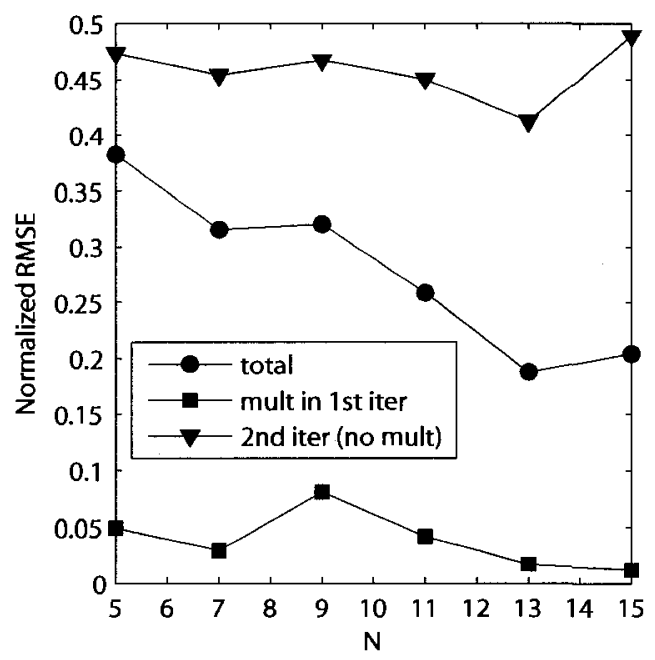

Figure 4.5: Normalized RMSE obtained after APIT is run with two iterations and a multilateration scheme as an intermediate step. $(-\bullet-)$ RMSE computed with the refined estimates and estimates of the 2nd iteration, (- -$)$ RMSE obtained by running multilateration on initial estimates, $(-\boldsymbol{\nabla}-)$ RMSE obtained by running APIT in the 2nd iteration. [DOI=0]. 


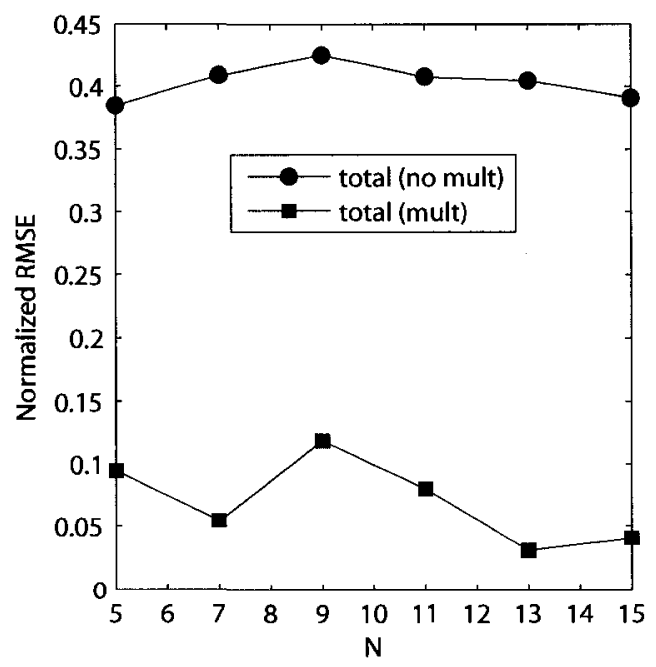

Figure 4.6: Normalized RMSE for two cases: $(-\bullet-)$ APIT is run with two iterations (- - ) APIT is run with two iterations and a multilateration scheme as a final step. $[\mathrm{DOI}=0]$.

One case consists of using both the anchors and the nodes with estimated location obtained in the first round as a new set of anchors in order to refine the remaining positions of the nodes. A second case consists of using only the $N$ sensors as anchors to refine the remaining estimates. This second case is the approach that we assume here.

Fig. 4.7 depicts the number of refined estimates. Note that not all nodes can be refined. The reason is that some of the $M$ sensors may not be within the range of at least three of the $N$ anchors. Therefore, their final estimates would be those obtained after APIT has been run twice. 


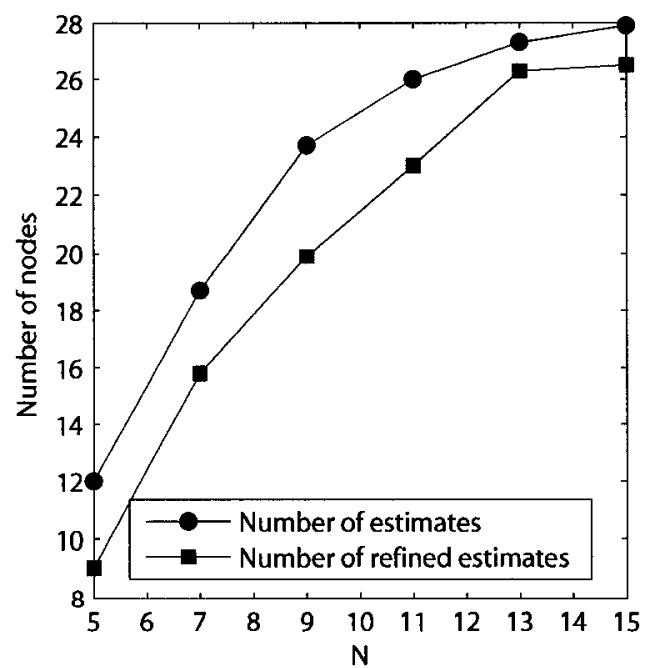

Figure 4.7: (-•-) Number of nodes with estimated location for Iterative APIT and (-口-) Number of refined estimates for Iterative APIT. [DOI=0].

\subsubsection{Performance of the enhanced algorithm in noisy en- vironments for sparse networks}

\section{Determination of nodes using Iterative APIT}

For the experiments in which we study the iterative procedure, we consider $M=$ 30 and $N$ varies from 5 to 15.

In a first experiment, we are interested on computing the number of nodes with estimated location when the Iterative APIT is run. The results are shown in Fig. 4.8. Note that a greates number of nodes can be estimated with the iterative procedure. Intuitively, one may think that even when increasing the level of noise, it is possible to estimate more nodes positions. This is validated in Fig. 4.9 . 


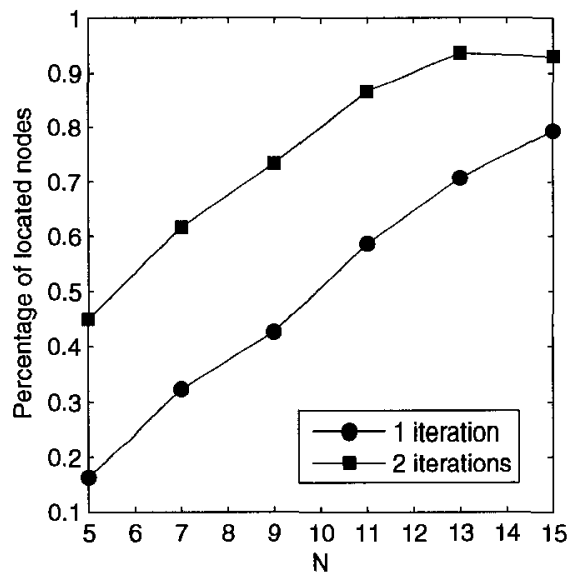

(a) $\mathrm{DOI}=0.1$

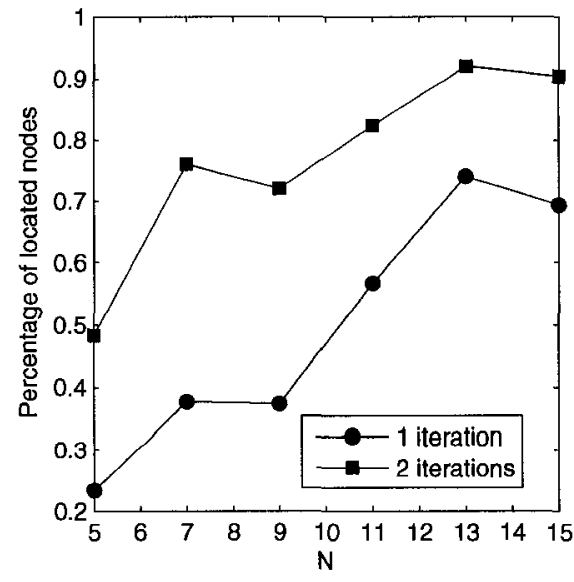

(b) $\mathrm{DOI}=0.3$

Figure 4.8: Comparison of located nodes when $(-\bullet-)$ APIT is run only once and $(-\mathbf{-}-)$ APIT is run with two iterations.[DOI $=0.1, \mathrm{DOI}=0.3]$

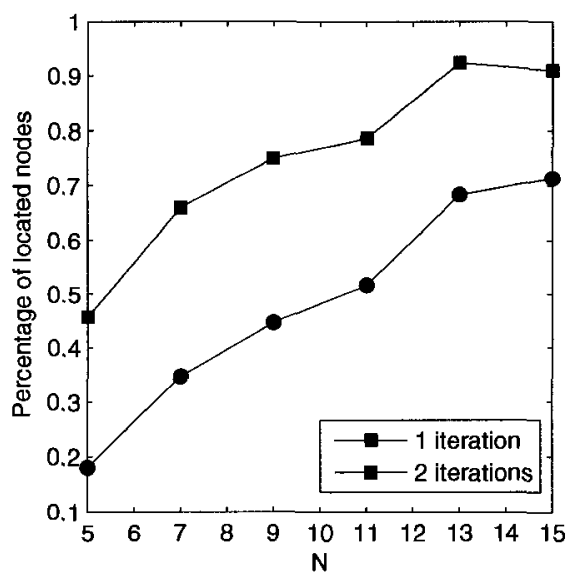

(a) $\mathrm{DOI}=0.5$

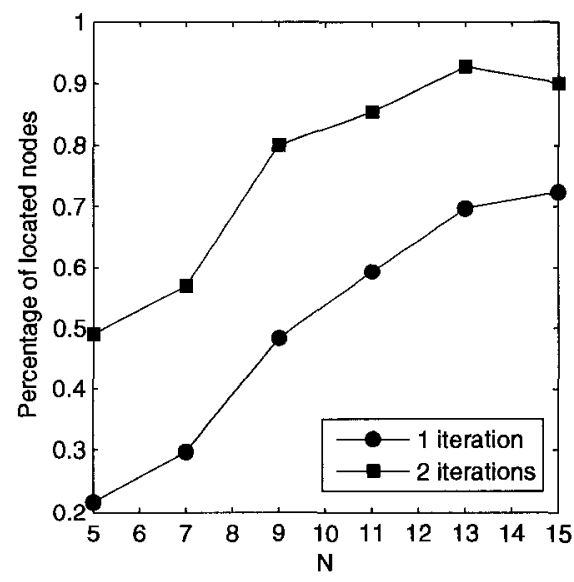

(b) $\mathrm{DOI}=0.7$

Figure 4.9: Comparison of located nodes when (- - ) APIT is run only once and $(-\square-)$ APIT is run with two iterations. [DOI $=0.5, \mathrm{DOI}=0.7]$ 


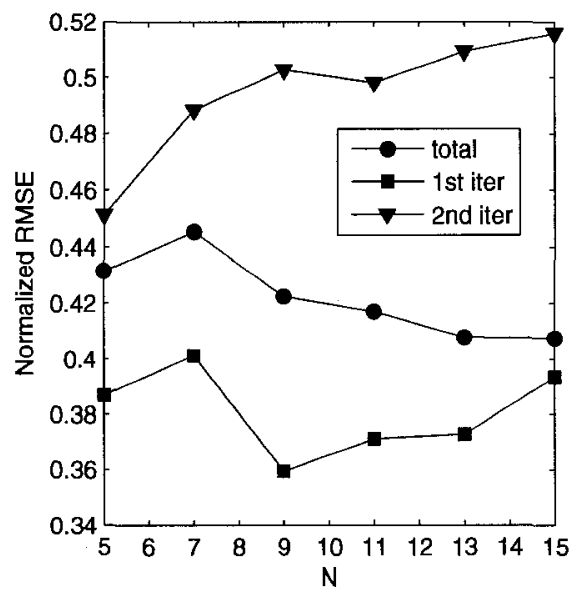

(a) $\mathrm{DOI}=0.1$

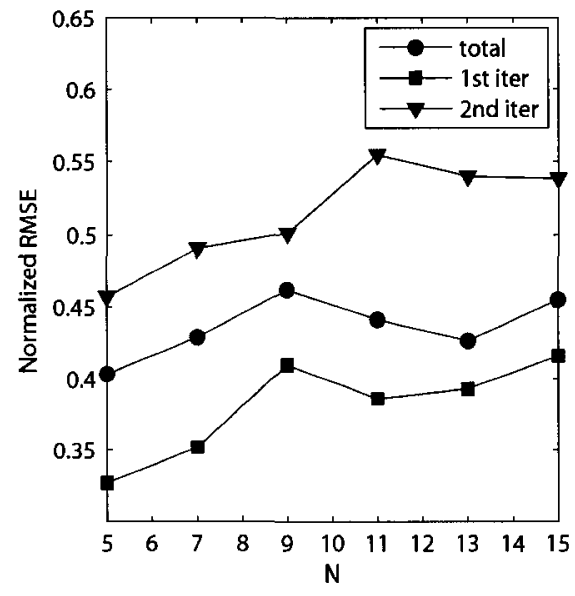

(b) $\mathrm{DOI}=0.3$

Figure 4.10: Normalized RMSE obtained after APIT is run with two iterations. (-•-) RMSE computed with the estimates obtained in two iterations, $(-\boldsymbol{\square}-)$ RMSE computed with the estimates obtained in the first iteration, $(-\boldsymbol{\nabla}-)$ RMSE computed with the estimates obtained in the second iteration. [DOI $=0.1$, $\mathrm{DOI}=0.3]$

\section{Location error introduced by Iterative APIT}

Another issue to investigate is how much the RMSE is affected when the APIT algorithm runs with two iterations in a noisy environment. Fig. 4.10 shows this scenario when $\mathrm{DOI}=0.1$ and $\mathrm{DOI}=0.3$. Note that the $R M S E$ computed with the estimates obtained in the two iterations [Fig. $4.10(-\bullet-)$ ] does not increase significantly in comparison with the RMSE computed with the estimates obtained in the first iteration [Fig. $4.10(-\mathbf{0}-)$ ]. We can arrive to the same conclusion when we increase the DOI values up to 0.5 and 0.7 . Fig. 4.11 shows this last case.

\section{Performance of the alternative test}

The APIT test proposed by [3] is an approach that requires to process data from the unknown sensor's neighborhood to decide if it is inside-outside a triangle 


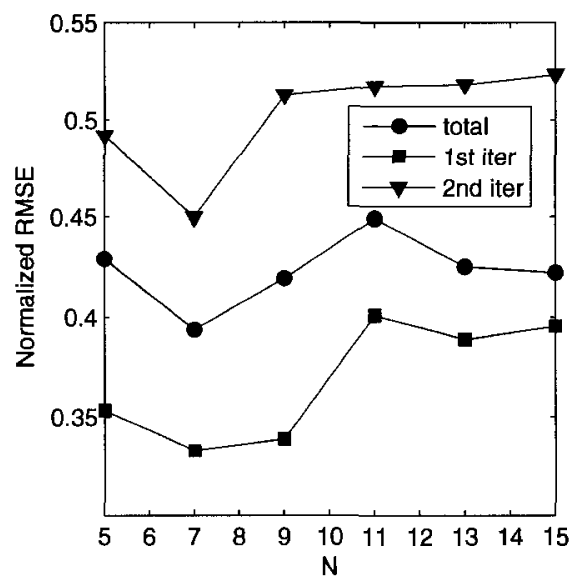

(a) $\mathrm{DOI}=0.5$

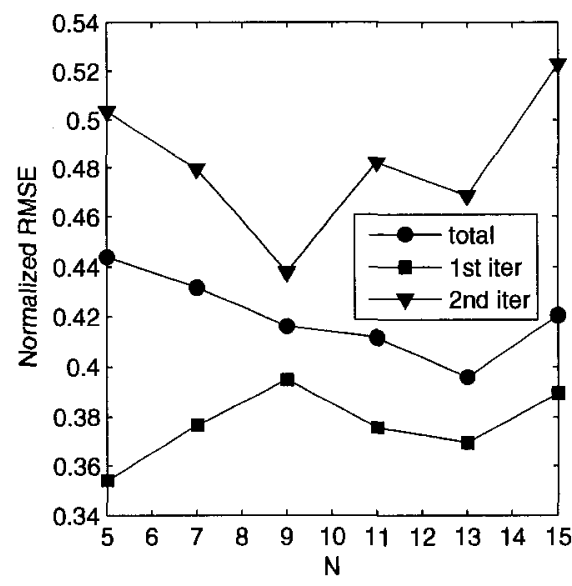

(b) $\mathrm{DOI}=0.7$

Figure 4.11: Normalized RMSE obtained after APIT is run with two iterations. (- - ) RMSE computed with the estimates obtained in two iterations, $(-\boldsymbol{-}-)$ RMSE computed with the estimates obtained in the first iteration, $(-\mathbf{\nabla}-)$ RMSE computed with the estimates obtained in the second iteration. [DOI $=0.5$, $\mathrm{DOI}=0.7]$

defined by three anchors. However, RF profiling can be used as an alternative method to make such a decision. The advantage of using RF profiling is that no neighbors are required and the number of wrong decisions is reduced. In order to validate our hypothesis we compare the errors obtained using the APIT test and those thrown by the RF profiling alternative test. A fully connected sparse network of $N=3$ is considered for this scenario. The length of the grid region is $0.5[m], \mathrm{DOI}=0.1$, and $M$ varies from 10 to 50 .

In order to evaluate the performance of the tests, we have to compare their decisions for an unknown sensor, with the correct inside-outside decision made for that sensor. The correct inside-outside decision can be obtained with the position of the unknown sensor.

For illustration purposes, consider the following example. Fig. 4.12(a) represents a decision made by the APIT test. Suppose that the APIT test decides that the unknown sensor $a$ is outside the triangle. Fig. 4.12(b) represents a decision made 


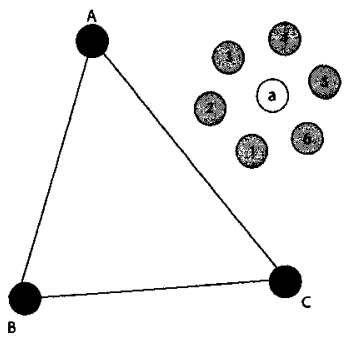

(a) APIT test

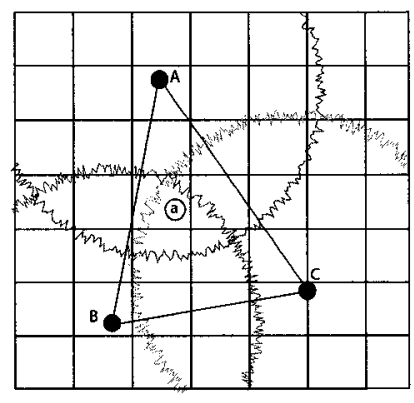

(b) Alternative Test

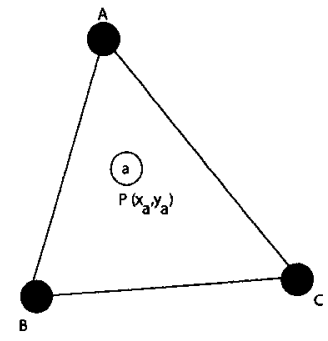

(c) Correct decision

Figure 4.12: The APIT test (a) and the Alternative Test (b) decide if the unknown sensor $a$ is inside or outside triangle A,B,C. Assuming the position of $a$ (c), we can know if $a$ is inside or outside the triangle.

by the alternative test. Such test decides that $a$ is inside the triangle. In order to know which test performed the wrong decision, we use the position of $a$ and the positions of $A, B, C$ in order to determine if it is inside or outside. For this case, it is revealed that $a$ is inside the triangle. Thus, the APIT test decided wrongly while the alternative test decided correctly. Note that Fig. 4.12(c) is used only for purposes of evaluating the performance of both tests.

Fig. 4.13 shows the ratio of wrong decisions introduced by each of the tests. It can be noticed how the proportion of wrong decisions introduced by the APIT test is higher than the one introduced by the alternative test. In other words, the alternative test outperforms APIT test in the proportion of accurate decisions.

Now, consider that we increase the level of noise up to $\mathrm{DOI}=0.7$. We repeat the same experiments as in the previous case. Fig. 4.14 denotes the proportion of wrong decisions introduced by each test separately.

Once again, the performance of the alternative test is better than the performance of the APIT test.

It should be mentioned that the APIT test depends not only on the size of the neighborhood of a node but also on the distribution of the nodes. Researchers 


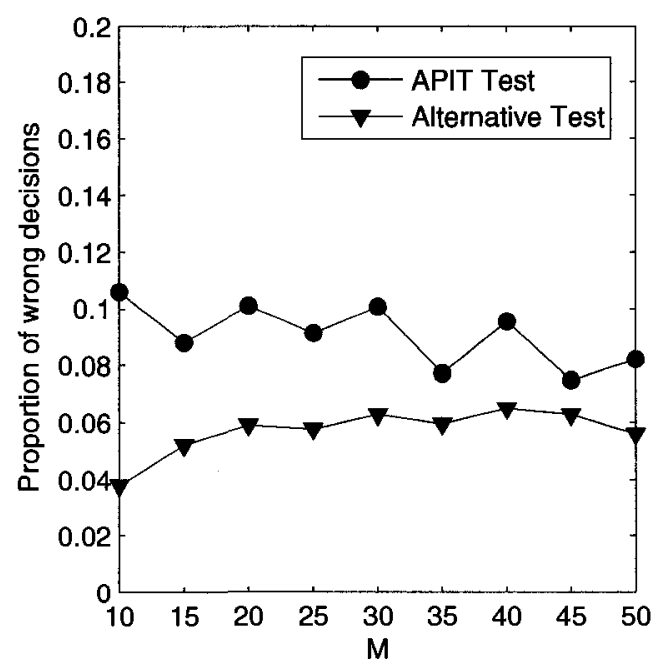

Figure 4.13: (- - -) Ratio of wrong decisions introduced by the APIT test and $(-\boldsymbol{\nabla}-)$ Ratio of wrong decisions introduced by the Alternative Test when $\mathrm{DOI}=0.1$

in [3] claim the success of their approach in very dense networks, in order to mitigate the irregular distribution of the sensors. Nevertheless, a solution was not provided for sparse networks.

We provide an alternative approach in these cases. Instead of relying on the distribution of the nodes in the neighborhood, it is preferable to collect $S S$ measurements at different points of a grid array, as illustrated in Fig. 4.12(b).

\section{Refinement as a final step for simple APIT}

APIT is a range-free scheme localization algorithm. The nodes positions obtained by running APIT can be further improved by the refinement mechanism even in noisy environments using range measurements. Certainly, these range measurements are affected by the noise in the system. However, it would be interesting to find out what is the interval of DOI values in which the refinement can help to improve the accuracy of the nodes positions.

It should be remembered that the refinement procedure proposed in Section 3.2.3 


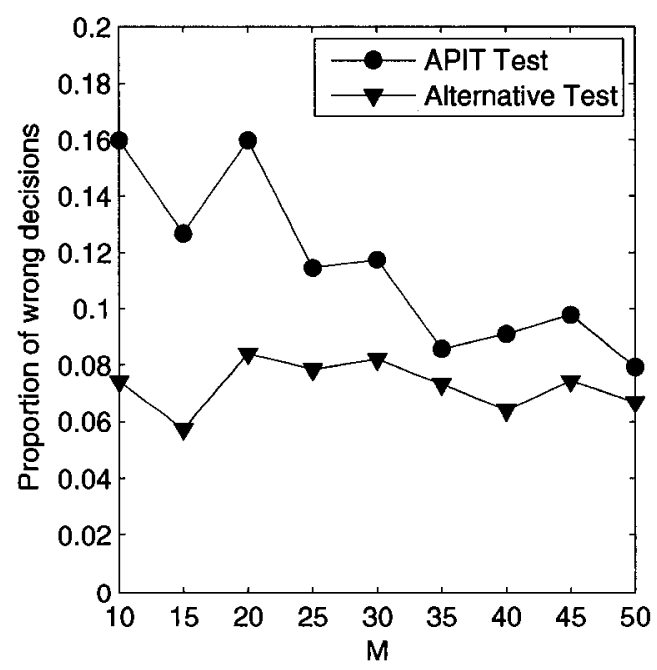

Figure 4.14: (- - -) Ratio of wrong decisions introduced by the APIT test and $(-\boldsymbol{\nabla}-)$ Ratio of wrong decisions introduced by the Alternative Test when $\mathrm{DOI}=0.7$

is an iterative mechanism. For the cases in which the iterative procedure fails to converge for a particular node, we maintain the initial position obtained by running APIT as our final node position.

The performance of the refinement in noisy environments is evaluated in sparse networks. $M=30$ unknown sensors are deployed randomly. Fig. 4.15 illustrates the results when $N=7$ anchors are deployed in the network. We selected this value of $N$ since it gives us an average number of audible anchors around 3 . If an unknown sensor listens to at least 3 anchors, then it is possible to apply the refinement procedure. It can be observed that for DOI values less than 0.1 , the location error is improved. Beyond this DOI value, the refinement does not contribute to reduce the location error. Indeed, the location error increases for DOI values greater than 0.1 . This means that the refinement procedure is sensitive to the noise in the system. However, refinement can be useful for scenarios in which range measurements are not very-noisy (i.e. range measurements for $\mathrm{DOI} \leq 0.1$ ). As we increase the DOI values, the range measurements between anchors and the 


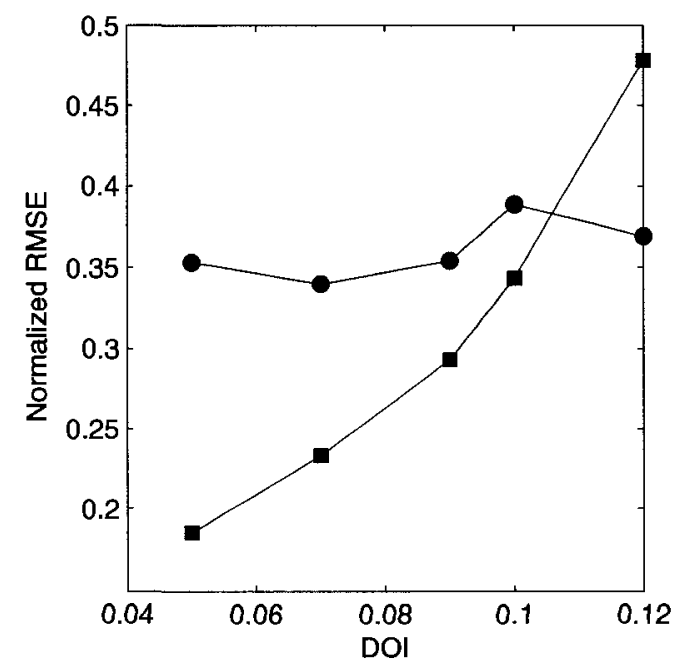

Figure 4.15: Refinement applied on APIT in a noisy environment. (- - -) Location error obtained by running APIT and (- -) Location error obtained by running the refinement scheme. $[\mathrm{N}=7]$

unknown sensors start to diverge from the true distances. Thus, even though we obtain an initial condition close to the true position, the refinement scheme will find a position that corresponds to the noisy range measurements.

\subsection{Performance in dense networks}

\subsubsection{Simulation Setup for dense networks}

\section{Placement Model}

For our experiments in dense networks, we consider two different placement models.

(1) Random distribution in regions. We divide the square of $10 \times 10\left[\mathrm{~m}^{2}\right]$ in four regions. In each region, we distribute $\frac{M}{4}$ unknown sensors and $\frac{N}{4}$ anchors randomly. We follow this methodology in order to increase the level of connectivity among nodes. Fig. 4.16 illustrates this scenario. 


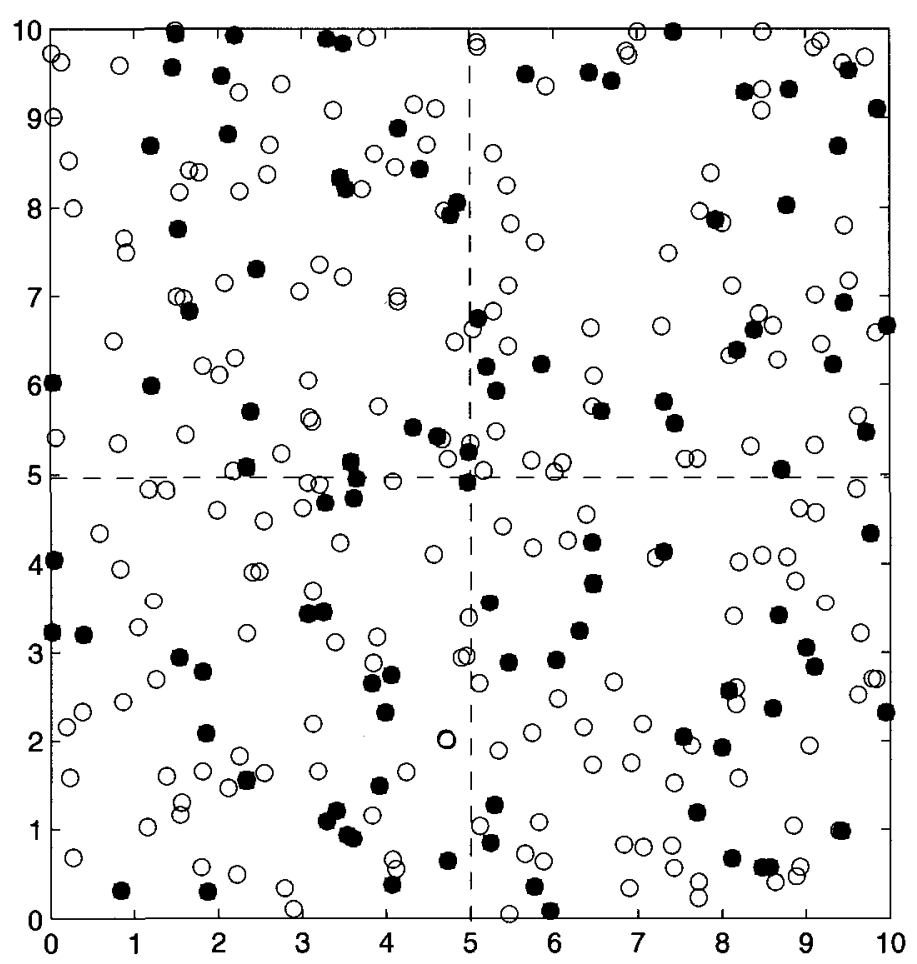

Figure 4.16: Dense Network with four regions. Nodes denoted as (ם) are anchors, nodes shown as (o) are unknown sensors.

(2) Deterministic distribution of anchors. A grid array divides the square of $10 \times 10\left[\mathrm{~m}^{2}\right]$ in regular regions, each one with a length of $1[\mathrm{~m}]$. A hundred anchors $(N=100)$ are deployed in the entire area in such way that the position of each anchor corresponds to one point of the grid array. Fig. 4.17 depicts this scenario.

\section{Non noisy environments}

For the experiments in which no noise is considered, the radio range $R$, is set to $1.5[\mathrm{~m}]$. The length of the grid side is set to $0.5[\mathrm{~m}]$ for the APIT Aggregation Step described in Section 2.2.2. We use confidence intervals of $95 \%$ for most of our results. 


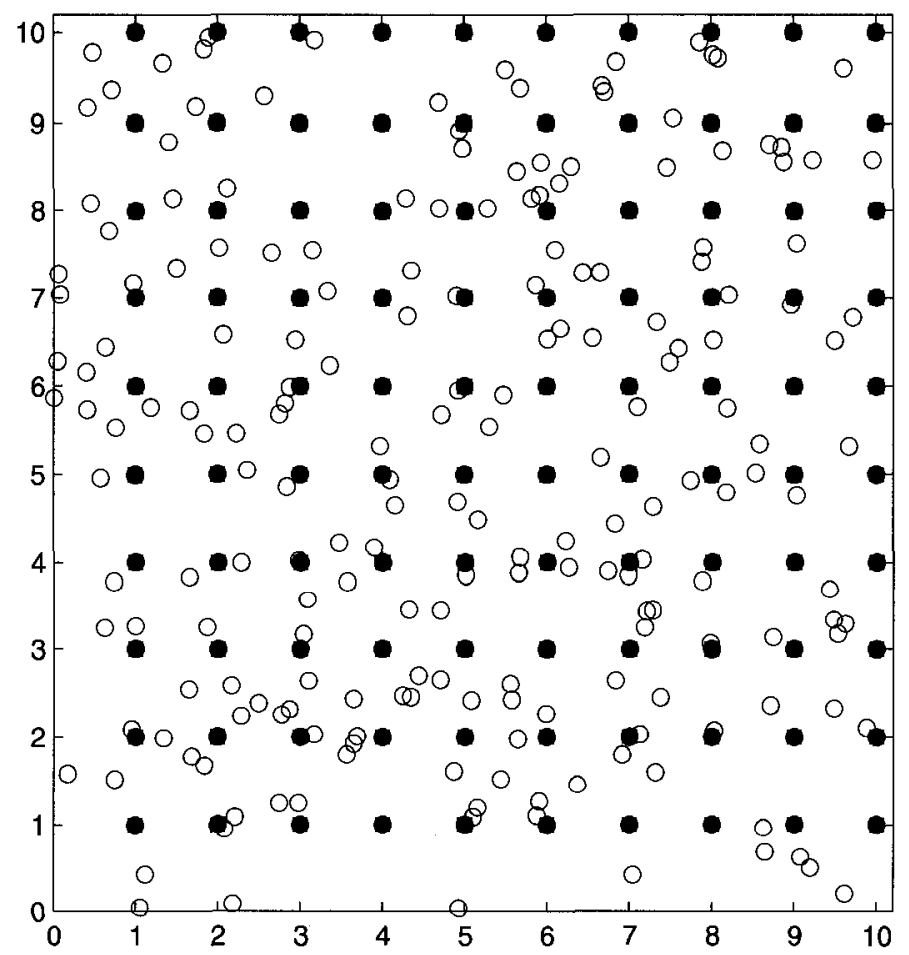

Figure 4.17: Dense Network in which anchors are deployed in each grid point. Nodes denoted as ( $)$ are anchors, nodes shown as (o) are unknown sensors.

\section{Noisy environments}

For the experiments in which noise is considered, the radio range $R$, is set to $1.5[m]$. We set the length of the grid side to $0.1 R$ (i.e. $0.15[m]$ ) for our evaluation of Iterative APIT and refinement scheme. This value of length of the grid side will allow us to not compromise the accuracy of the location estimation. For the evaluation of the performance of the alternative test, the length of the grid side is set to $0.5[\mathrm{~m}]$

Similarly to the case of non noisy enviroments, we use confidence intervals of $95 \%$ for most of our results. 


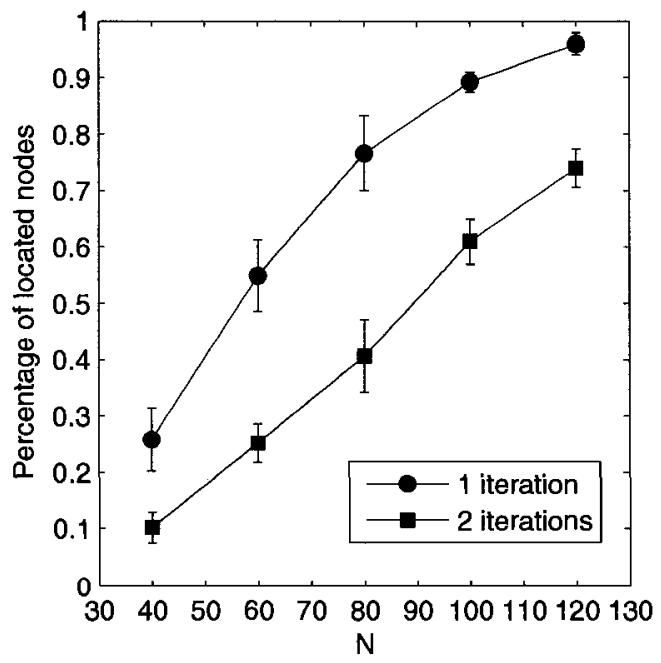

Figure 4.18: (-口-) Number of nodes estimated in one iteration and $(-\bullet-)$ Number of nodes estimated in two iterations. [DOI=0]

\subsubsection{Performance of the enhanced algorithm in non noisy environments for dense networks}

\section{Determination of nodes using Iterative APIT}

For the following experiments, the deployment of the sensors follows the Random distribution in regions setup described in Section 4.5.1. In a first experiment, $M=200$ unknown sensors are deployed in our square field. The number of anchors $N$ varies from 40 to 120 .

From our results in sparse networks, we observed that more nodes positions could be estimated using our iterative scheme. Now, we applied the same methodology on dense networks. The first set of nodes positions and anchors is used as a new set of anchors in order to estimate a second set of nodes positions. Fig. 4.18. shows that after two iterations, more node positions are estimated. 


\section{Location error introduced by Iterative APIT}

In a second experiment we are interested in analyzing how the location error is affected when Iterative APIT is applied on dense networks. The same conditions in the number of anchors and unknown anchors are considered. We use the APIT test as the approach to decide if an unknown sensor is inside or outside any triangle defined by its audible anchors. Some important observations should be mentioned from Fig. 4.19:

First, the location error after running two iterations increases in comparison with the location error obtained in the first iteration. Refer to Fig. $4.19[(-\bullet-)$ and $(-\mathbf{\square})]$. Nevertheless, the increase is not much significant.

Second, even increasing the number of anchors on the field does not necessarily reduce the location error using the Random distribution in regions setup. When the number of anchors is increased, the average number of anchors listened to by an unknown sensor increases. However, the number of wrong decisions performed by the unknown sensors on choosing whether they are inside or outside the triangles defined by the anchors can also increase. This would increase the location error of the system [Fig .4.19 (- -$)]$. The probability that a wrong decision occurs depends on: 1) the number of unknown sensors deployed and 2) the distribution of the unknown sensors. As observed in Fig. 4.16 we have increased the density of the unknown sensors in comparison to the density of the unknown sensors in a sparse network. However, the distribution of the unknown sensors on the field is still irregular.

Third, when the number of anchors $N$ increases, more nodes positions are estimated with simple APIT. A similar situation occurs for our iterative scheme. One disadvantage of this methodology, is that the location error increases. We are estimating a smaller number of nodes positions in the second round and we 


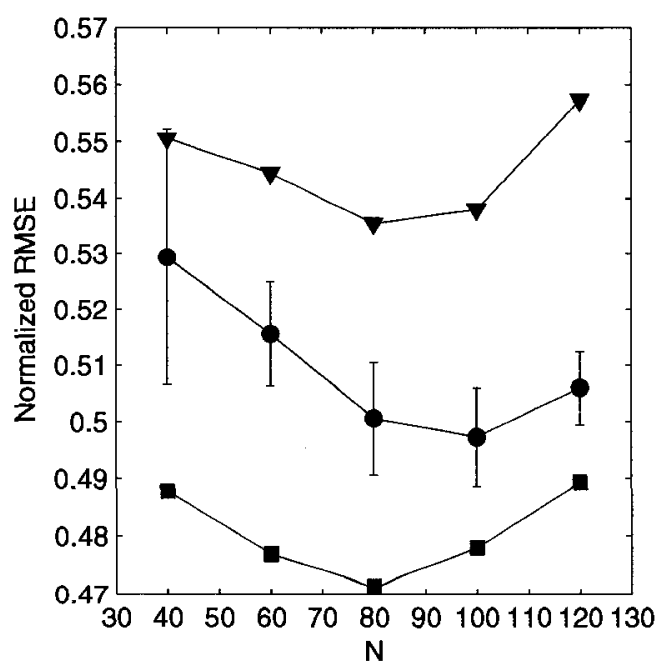

Figure 4.19: Normalized RMSE obtained after APIT is run with two iterations. $(-\bullet-)$ RMSE computed with the estimates obtained in two iterations, $(-\boldsymbol{\square}-)$ RMSE computed with the estimates obtained in the first iteration, (- -$)$ RMSE computed with the estimates obtained in the second iteration. [DOI $=0$ ]

are using more nodes estimates obtained in the first round as anchors.

As an example, consider the case when $N=120$ in Fig. 4.18. The percentage of unknown sensors estimated is $74 \%$ when simple APIT is run. For Iterative APIT, we increase the percentage up to $96 \%$. Thus, we estimated $22 \%$ out of the $M$ unknown sensors in the second iteration. Since the $74 \%$ of the $M$ sensors along with the $N$ anchors are used as a new set of anchors for the estimation of the $22 \%$ of the nodes, we can expect that the location error in the second iteration would increase. In Fig. 4.19, it is shown that the location error is close to $56 \%$ of the radio range. From the first to the second iteration, it increases $0.07 R$. However, the increment of the location error obtained from the two iterations [Fig. 4.19 $(-\bullet-)$ ] compared with the location error of the first iteration [Fig. $4.19(-\mathbf{-}-)$ ] is less than $0.02 R$. 


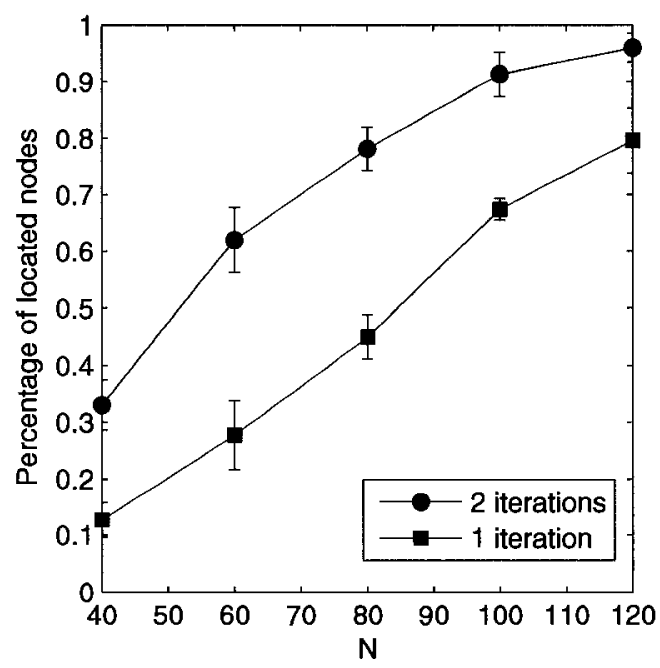

Figure 4.20: (- - -) Number of nodes estimated in one iteration and (- $\bullet-)$ Number of nodes estimated in two iterations. [DOI=0.1]

\subsubsection{Performance of the enhanced algorithm in noisy en- vironments for dense networks}

\section{Determination of nodes using Iterative APIT}

Even in the presence of noise, Iterative APIT is able to estimate a greater number of nodes positions. For this experiment, we consider $M=200$ and $N$ varies from 40 to 120 . Our results are shown in Fig. 4.20 when DOI=0.1.

\section{Location error introduced by Iterative APIT}

For the following experiments, we maintain the APIT test as the approach to decide if an unknown sensor is inside or outside any triangle defined by its audible anchors.

\section{(1) DOI is fixed and the number of anchors varies}

The location error behavior is evaluated when the number of anchors $N$ varies and the level of noise DOI is fixed. We maintain the number of un- 
known sensors $M$ fixed to 200 . The number of anchors, $N$, varies from 40 to 120. Likewise, the deployment of the sensors follows the Random distribution in regions setup.

We mentioned previously that increasing the number of anchors in a field can increase the location error of the system if the unknown sensors are positioned irregularly in the neighborhoods of the unknown sensors. This can be observed in Fig. $4.21\left[(-\mathbf{\nabla}-)\right.$ and $\left.\left(-\mathbf{-}_{-}\right)\right]$. For some values of $N$, the location error does not decrease. However, it should be noticed that the location error obtained with the nodes positions of the two iterations does not vary significantly. It lies between the $57 \%$ and the $60 \%$ of the radio range [Fig. $4.21(-\bullet-)$ ], for all values of $N$.

It would be interesting to evaluate the location error obtained in two iterations for denser networks, in order to have a less irregular distribution of the nodes. One may think that by increasing the number of anchors for this scenario the location error would decrease since less wrong decisions from the APIT test would be performed. We have left this as future work.

\section{(2) The number of anchors is fixed and DOI varies}

For this experiment, we study the case in which we increase the level of noise and how this affects the location error in the iterative algorithm. The number of unknown sensors $M$ is set to 200 and the number of anchors $N$ is set to 100 . The deployment of the sensors follows the Random distribution in regions setup.

For each DOI value, we compare the location error obtained from running simple APIT and the location error obtained from running Iterative APIT. Fig. 4.22 shows our results using confidence intervals of $95 \%$.

It can be noticed how the location error increases on average for both sim- 


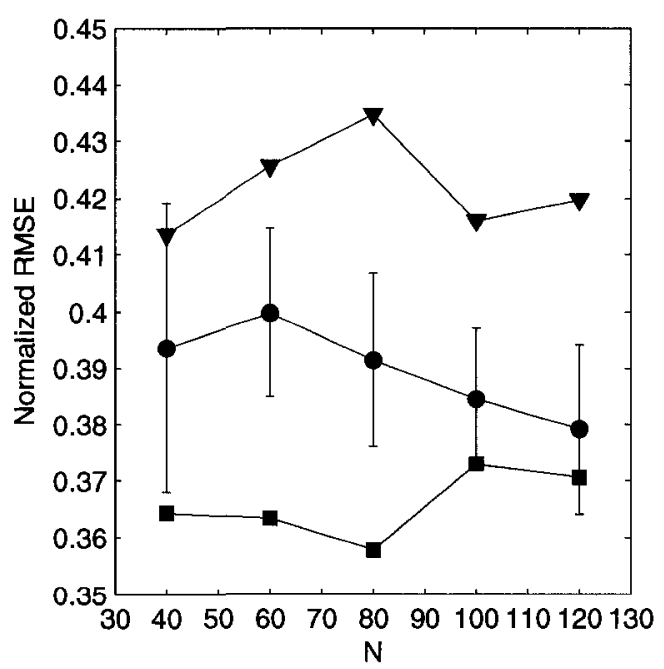

Figure 4.21: Normalized RMSE obtained after APIT is run with two iterations. $(-\bullet-)$ RMSE computed with the estimates obtained in two iterations, (- - -) RMSE computed with the estimates obtained in the first iteration, (-v-) RMSE computed with the estimates obtained in the second iteration. [DOI $=0.1]$

ple APIT and Iterative APIT. However, the difference of location errors between simple APIT and Iterative APIT is not significant. This shows that our proposed iterative algorithm is robust for different degrees of noisy environments since it is based on the foundations of APIT. Researches in [3] observed this situation for APIT and claimed that the APIT Aggregation Step helps not to increase significantly the location error for different levels of noise. Likewise, we compute the number of nodes positions for both simple APIT and Iterative APIT. This is shown in Fig. 4.23. Although the location error increases with Iterative APIT in Fig. 4.22, the number of nodes positions is not compromised. Moreover, we estimate more nodes positions with Iterative APIT.

\section{(3) Performance of Iterative APIT for two placement models}

In this experiment we are interested on analyzing how the location error improves when the anchors are deployed uniformly through the field using 


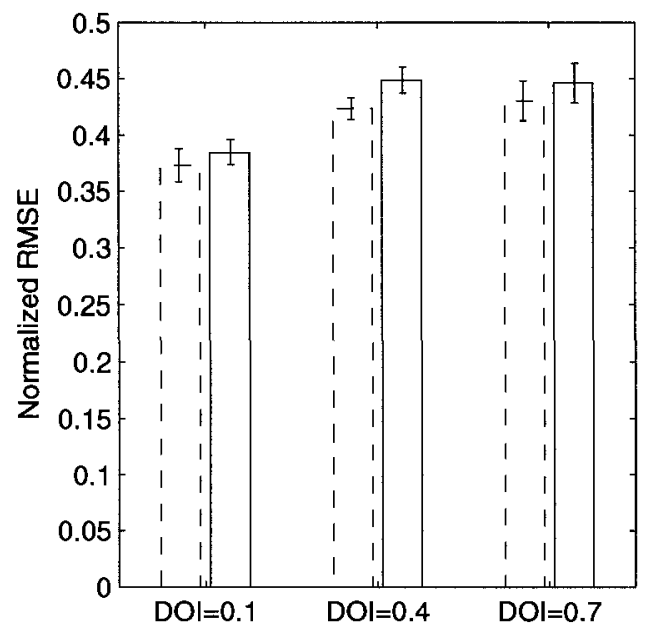

Figure 4.22: (--) Location error using simple APIT and (-) Location error using our iterative scheme.

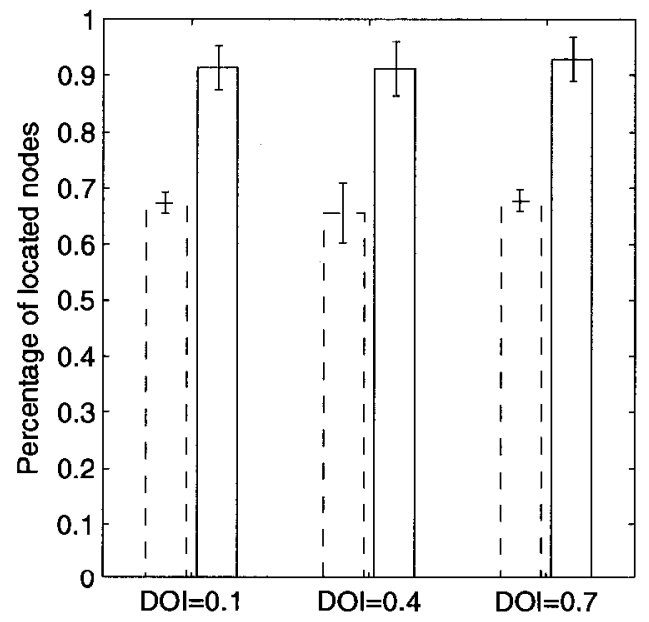

Figure 4.23: (--) Ratio of nodes estimates using simple APIT and (-) Ratio of nodes estimates using our iterative scheme. 


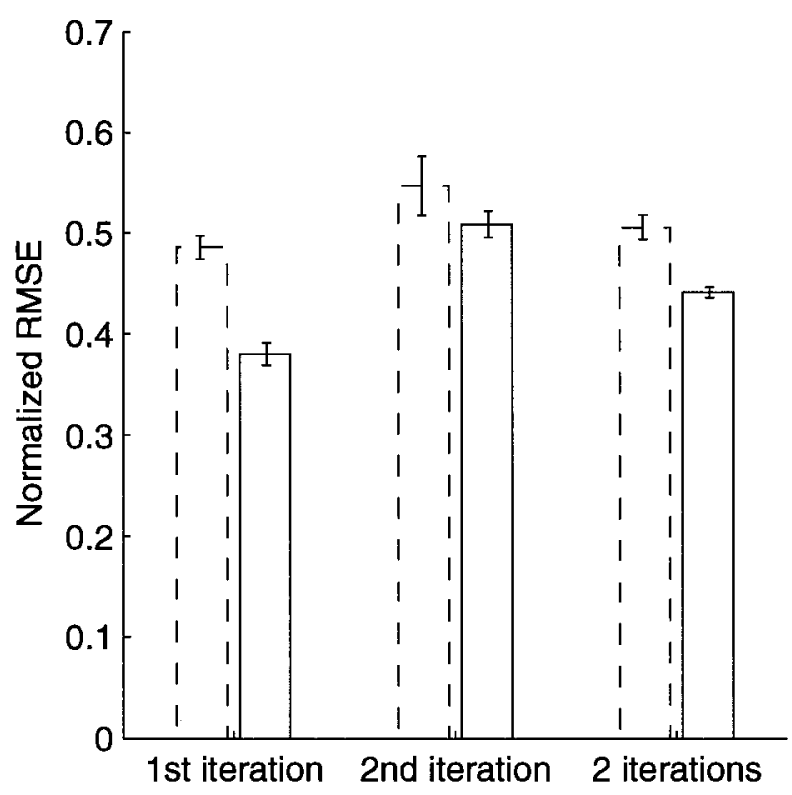

Figure 4.24: (--) Location error obtained when anchors are distributed randomly and ( - ) Location error obtained when anchors are deployed deterministically. $[\mathrm{DOI}=0.1]$

the Deterministic distribution of anchors setup. The level of noise DOI is set to 0.1 . Additionally, we consider $M=200$ and $N=100$.

The location error obtained in this system is compared with the one obtained when the anchors are distributed among the four regions. We compute the $95 \%$ confidence interval in our results plotted in Fig. 4.24. It can be observed how the location error decreases when the the anchors are distributed in specific locations. The location error is grouped under three cases: 1) location error obtained with the first set of nodes estimates, 2) location error obtained with the second set of nodes estimates, and 3) location error obtained with the total number of nodes estimates.

The next study is in terms of the number of estimated nodes using simple APIT and our iterative scheme. We compute the number of nodes positions using the Deterministic distribution of anchors setup and the number 


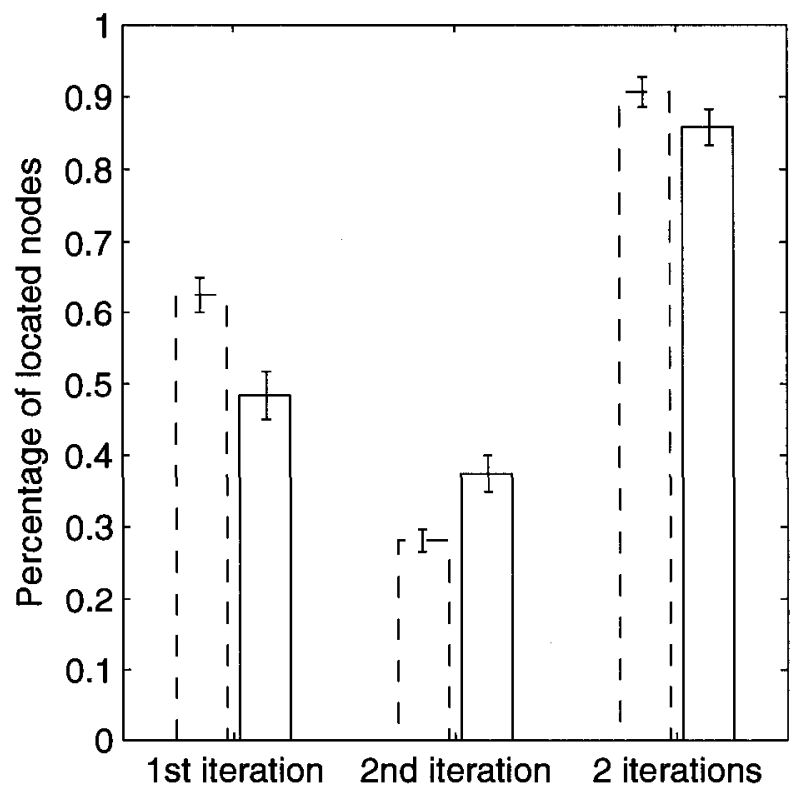

Figure 4.25: (--) Ratio of nodes estimates obtained when anchors are distributed randomly and (-) Ratio of nodes estimates when anchors are deployed deterministically. $[\mathrm{DOI}=0.1]$

of nodes positions using the Random distribution of anchors setup. Fig. 4.25 shows the results of this experiment with a $95 \%$ confidence interval. Observe the third pair of bars in Fig. 4.25. The algorithm using the deterministic deployment of anchors estimates in average less nodes positions than the algorithm using the random distribution of anchors.

Distributing the anchors deterministically on the field allows us to define uniform triangular regions. However, this does not necessarily mean that the number of estimated nodes positions should be increased. On the other hand, the difference on the estimated number of nodes positions using both schemes is not significant. The scheme based on the deterministic distribution of anchors estimates $90 \%$ of the unknown sensors, while the scheme based on the random distribution of anchors estimates around $85 \%$ of the unknown sensors. 


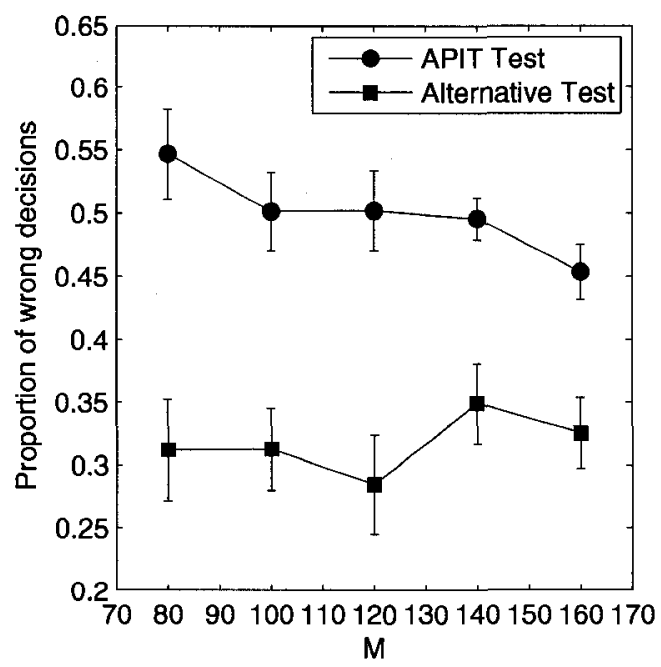

Figure 4.26: Performance of the tests in a dense network. (- $\bullet-)$ Ratio of wrong decisions introduced by the APIT test and ( $-\boldsymbol{\nabla}-)$ Ratio of wrong decisions introduced by the Alternative Test when DOI $=0.1$.

\section{Performance of the alternative test}

For the evaluation of the performance of the alternative test, the length of the grid side is set to $0.5[\mathrm{~m}]$. As a metric for this evaluation we compute the proportion of wrong decisions introduced by our approach. The proportion of wrong decisions is obtained through the failed decisions made by the unknown sensors when they have to choose whether they are inside or outside the triangles defined by their audible anchors.

Similarly to the case of sparse networks, we evaluate the performance of the alternative test, but now in dense networks. The distribution of the sensors follows the Random distribution in regions setup. We set the number of anchors $N$ to 40 . The number of unknown sensors $M$ varies from 80 to 160 . Fig. 4.26 denotes the proportion of wrong decisions when the unknown sensors choose whether they are inside-outside the triangles for the two tests when DOI=0.1. After obtaining these results, we can mention the following. 
First, the proportion of wrong decisions using the APIT test is always greater than the one using the alternative Test. This means that the alternative test outperforms the APIT test.

Second, the radio range of the sensors affects both the APIT test and the alternative test in the proportion of wrong decisions. Particularly, the proportion of wrong decisions introduced by each test depends on the following,

(1) Proportion of wrong decisions for the APIT test. The number of SS measurements collected from the neighbors of an unknown sensor will depend on the density of the sensors and the radio range $R$. Furthermore, the high proportion of wrong decisions can be attributed to the irregular distribution of the unknown sensors.

(2) Proportion of wrong decisions for the alternative test. The radio range $R$ determines the number of points of the grid for which we can collect triplets of SS measurements. Furthermore, the granularity of the grid contributes to its performance. When the length of the grid side decreases, more points of the grid array that lie inside the radio range can be evaluated, thus improving the accuracy of its decisions.

Similar observations can be made when the performance of the alternative test is evaluated for DOI=0.7. The results are shown in Fig. 4.27.

\section{Refinement as a final step for simple APIT}

Finally, we evaluate the refinement scheme for dense networks in noisy environments. We assume a scenario in which $N=40$ anchors and $M=200$ unknown 


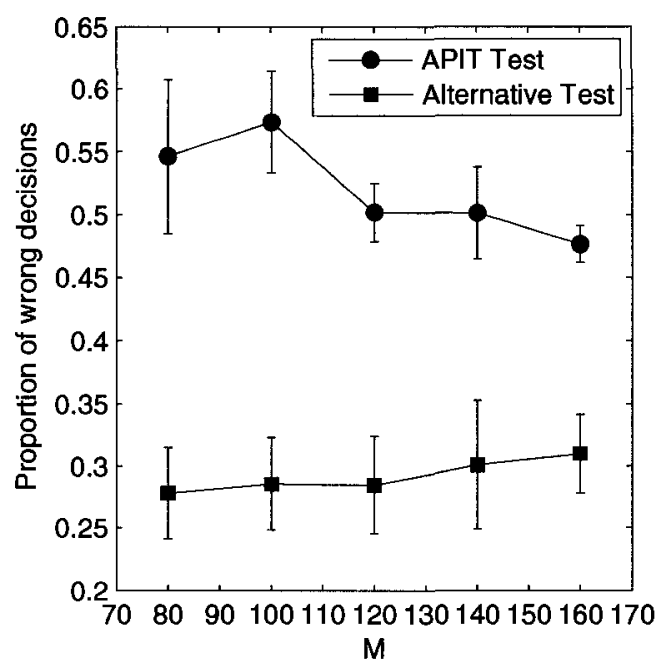

Figure 4.27: Performance of the tests in a dense network. (- $\bullet$ ) Ratio of wrong decisions introduced by the APIT test and $(-\mathbf{\nabla}-)$ Ratio of wrong decisions introduced by the Alternative Test when $\mathrm{DOI}=0.7$.

sensors are deployed using the Random distribution in regions setup.

Similarly to the case of sparse networks, we are interested on finding the interval of DOI values in which the refinement scheme improves the accuracy of the nodes positions calculated. Fig. 4.28 shows our results. Note that we have increased the number of anchors in comparison to the number of anchors we employed for the evaluation of the refinement scheme in sparse networks. From our experiments we computed the average number of anchors listened to by an unknown sensor for the scenario of dense networks. An average number of audible anchors around 3 was obtained. This is attributed to the fact that we are using a small value for the radio range $(R=1.5[\mathrm{~m}])$ in this experiment. Since each unknown sensor has few audible anchors, it is reasonable to mention that the refinement scheme can reduce the location error for a small interval of DOI values. For DOI values less than 0.16 , the refinement procedure helps to improve the accuracy of the nodes positions calculated. 


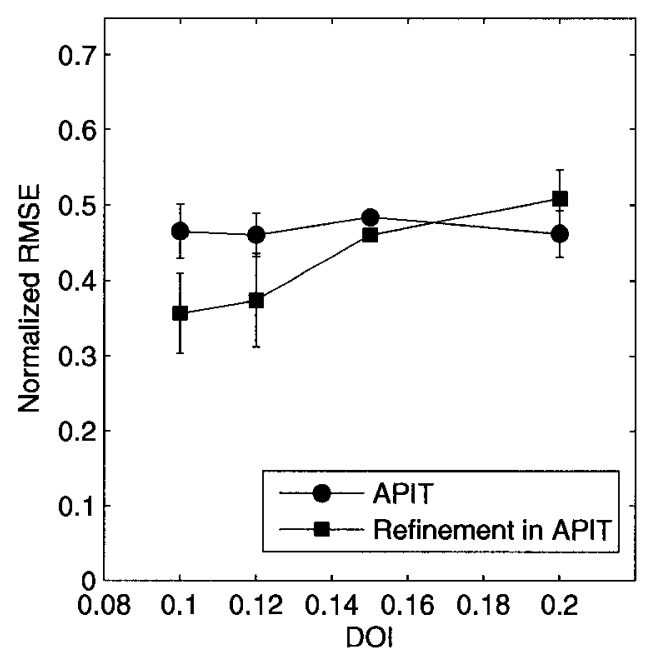

Figure 4.28: Refinement applied on APIT in a noisy environment. (- $\bullet-)$ Location error obtained by running APIT and (-ם-) Location error obtained by running the refinement scheme. $[\mathrm{N}=40]$ 


\section{Chapter 5}

\section{Conclusions and Proposed}

\section{Research}

Based on our approach for localization in wireless sensor networks and our results, we present our conclusions and propose future research.

\subsection{Conclusions}

This thesis has presented a hybrid localization algorithm which is based on advanced range-free localization couple to range-based enhancement. It is robust in noisy environments due to the acquisition of range measurements from the anchors that are in direct communication with the unknown sensors. Therefore, our approach is a hybrid algorithm that combines the advantages of both range-free and range-based schemes.

Specifically, these advantages are the following,

(1) Our approach shows a reasonably good performance in non noisy and noisy environments. 
(2) Furthermore, the enhanced algorithm is able to compute more node positions thanks to a simple and efficient iterative method. If we assume two identical scenarios in which one runs simple APIT and the second one runs Iterative APIT, Iterative APIT is able to estimate more nodes positions

(3) It was shown that the iterative methodology does not increase the location error significantly. We have shown this through different scenarios in which the number of anchors and the DOI values vary. The location error is studied when the number of anchors are distributed either randomly or deterministically for our iterative scheme. The location error decreases for the latter without compromising the estimated number of nodes positions.

(4) The location error introduced by the estimation of the nodes positions can be reduced using a refinement scheme. The refinement scheme (i.e. multilateration) can be applied on either simple APIT or our iterative method. Multilateration has been applied for other problems in the literature [6]. In our approach, we use it in order to improve the accuracy of the estimations obtained either from simple APIT or Iterative APIT. Likewise, we studied for which DOI values the refinement scheme is helpful for both sparse and dense networks.

(5) Simulation results show us that each of the enhancements proposed (i.e. the refinement scheme, the iterative methodology and the alternative test) provides a reasonably good performance in sparse and dense networks. 


\subsection{Future work}

In the following lines, we discuss the future work in the challenging field of localization in sensor networks.

(1) Even though we evaluated the performance of our enhanced algorithm in dense networks (i.e. networks of around hundreds of sensors), we did not evaluate it on large-scale networks (i.e. networks with thousands of sensors). Thus, the same set of simulations presented on this thesis can be run for these type of networks.

(2) It would be interesting to propose a mathematical analysis for our enhanced algorithm. Specifically, the refinement procedure requires further study. We implemented a simple least squares minimization. However, previous works such as [6], presented a multilateration scheme in which uncertainities are assigned to range-measurements in order to give more reliability to those that are not heavily affected by noise.

(3) The scalability of our iterative scheme should be studied with respect to the associated computational complexity.

(4) Our simulations were run in environments with no obstacles. However, a real scenario may have obstacles affecting the connectivity among sensors. The analysis on these type of environments would provide some insights into defining withch sensors will be assigned as anchors.

(5) Our enhanced algorithm has a good performance on two dimensional scenarios. Extrapolating our approach to three-dimensional topologies would extend the reliability of its performance in real scenarios. 
(6) We have obtained through simulations the location error given the number of anchors $N$ and the number of unknown sensors $M$. However, some applications would need to obtain which values of $N$ and $M$ are required for a given value of location error and a particular noisy environment.

(7) Our approach is a single-hop algorithm. One drawback of this scheme is that an unknown sensor needs to be in connectivity with many anchors in order to estimate its position. A further study would be to extrapolate our enhancements so that it can be run in a multi-hop fashion. 


\section{References}

[1] F. Martincic and L. Schwiebert, Handbook of Sensor Networks: Algorithms and Architectures. Wiley Series on Parallel and Distributed Computing, 2005, ch. Introduction to Wireless Sensor Networking, pp. 1-40.

[2] J. Bachrach and C. Taylor, Handbook of Sensor Networks: Algorithms and Architectures. Wiley Series on Parallel and Distributed Computing, 2005, ch. Localization in Sensor Networks, pp. 327-349.

[3] T. He, C. Huang, B. Blum, J. Stankovic, and T. Abdelzaher, "Range-free localization schemes for large scale sensor networks," in Proceedings of the ninth annual international conference on Mobile computing and networking (MobiCom 2003), San Diego, California, Sept. 2003, pp. 81-95.

[4] N. Bulusu, J. Heidemann, and D. Estrin, "GPS-less low cost outdoor localization for very small devices," IEEE Personal Communications Magazine, pp. 28-34, Oct. 2000.

[5] C. Liu and K. Wu, "Performance evaluation of range-free localization methods for wireless sensor networks," in Performance, Computing, and Communications Conference (IPCCC '05), Phoenix, AZ, 2005, pp. 59-66. 
[6] D. Niculescu and B. Nath, "Dv based positioning in ad hoc networks," In Journal of Telecommunication Systems, vol. 22, pp. 267-280(14), Jan. 2003.

[7] R. Nagal, H. Shrobe, and J. Bachrach, "Organizing a global coordinate system from local information on an ad hoc sensor network," in The Second International Workshop on Information Processing in Sensor Networks (IPSN '03), Palo Alto, Apr. 2003, pp. 333-348.

[8] D. Moore, J. Leonard, D. Rus, and S. Teller, "Robust distributed network localization with noisy range measurements," in Proceedings of ACM Sensys04, Baltimore, MD, Nov. 2004, pp. 50-61.

[9] J. Costa, N. Patwari, and A. Hero, "Distributed weighted multidimensional scaling for node localization in sensor networks," ACM Transactions on Sensor Networks, vol. 2, pp. 39-64, Feb. 2006.

[10] K. Whitehouse and D. Culler, "A robustness analysis of multi-hop rangingbased localization approximations," in The Fifth International Conference on Information Processing in Sensor Networks (IPSN '06), Nashville, TN, Apr. 2006, pp. 317-325.

[11] N. Patwari, J. Ash, S. Kyperountas, A. O. Hero, R. M. Moses, and N. S. Correal, "Locating the nodes: Cooperative localization in wireless sensor networks," IEEE Signal Processing Mag., vol. 22, pp. 54-69, July 2005.

[12] N. Patwari, A. O. Hero, M. Perkins, N. S. Correal, and R. J.O'Dea, "Relative location estimation in wireless sensor networks," IEEE Trans. Signal Processing, vol. 8, pp. 2137-2148, Aug. 2003.

[13] N. Patwari. (2006, Oct.) Sensor network measurements. [Online]. Available: http://www.ece.utah.edu/ npatwari/ 
[14] N. Patwari and A. Hero, "Using proximity and quantized RSS for sensor localization in wireless networks," in Proceedings of the 2nd Internation ACM Workshop on Wireless Sensor Networks and Applications (WSNA 03), San Diego,California, Sept. 2003, pp. 20-29.

[15] N. Patwari, A. O. Hero, and J. A. Costa, Secure Localization and Time Synchronization for Wireless Sensor and Ad Hoc Networks. Springer, Nov. 2006, vol. 30, ch. Learning Sensor Location from Signal Strength and Connectivity, pp. 57-81.

[16] G. Zhou, T. He, S. Krishnamurthy, and J. A. Stankovic, "Models and solutions for radio irregularity in wireless sensor networks," ACM Transactions on Sensor Networks, pp. 221-262, May 2006.

[17] K. Whitehouse, "The design of calamari: an ad-hoc localization system for sensor networks," Master's thesis, University of California at Berkeley, 2002.

[18] K. Whitehouse, C. Karlof, A. Woo, F. Jiang, and D. Culler, "The effects of ranging noise on multihop localization: an empirical study," in The Fourth International Conference on Information Processing in Sensor Networks (IPSN '05), Los Angeles, California, Apr. 2005, pp. 73-80.

[19] P. Bahl and V. N. Padmanabhan, "RADAR: an in-building RF-based user location and tracking system," in INFOCOM 2000, Tel Aviv, Israel, Mar. 2000, pp. $775-784$.

[20] C. Savarese, J.Rabaey, and K. Langendoen, "Robust positioning algorithms for distributed ad-hoc wireless sensor networks," in USENIX Technical Annual Conference, Monterey, California, June 2002, pp. 317-327. 
[21] Sharcnet (Shared Hierarchical Academic Research Computing Network). [Online]. Available: http://www.sharcnet.ca 